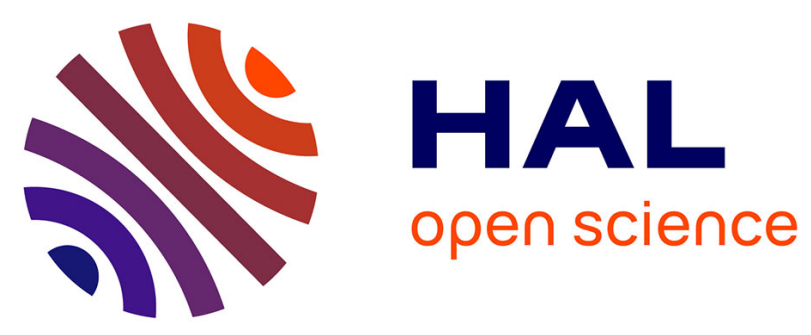

\title{
Characterisation and propagation of spatial fields in deterioration models: application to concrete carbonation
}

Ndrianary Rakotovao Ravahatra, Emilio Bastidas-Arteaga, Franck Schoefs, Frederic Duprat, Thomas de Larrard, M. Oumouni

\section{To cite this version:}

Ndrianary Rakotovao Ravahatra, Emilio Bastidas-Arteaga, Franck Schoefs, Frederic Duprat, Thomas de Larrard, et al.. Characterisation and propagation of spatial fields in deterioration models: application to concrete carbonation. European Journal of Environmental and Civil Engineering, 2019, pp.1-27. 10.1080/19648189.2019.1620133 . hal-02146486

\section{HAL Id: hal-02146486 \\ https://hal.science/hal-02146486}

Submitted on 4 Jun 2019

HAL is a multi-disciplinary open access archive for the deposit and dissemination of scientific research documents, whether they are published or not. The documents may come from teaching and research institutions in France or abroad, or from public or private research centers.
L'archive ouverte pluridisciplinaire HAL, est destinée au dépôt et à la diffusion de documents scientifiques de niveau recherche, publiés ou non, émanant des établissements d'enseignement et de recherche français ou étrangers, des laboratoires publics ou privés. 


\title{
Characterisation and propagation of spatial fields in deterioration models: application to concrete carbonation
}

\author{
N. Rakotovao Ravahatra ${ }^{a}{ }^{*}$, E. Bastidas-Arteaga ${ }^{\mathrm{b}}$, F. Schoefs ${ }^{\mathrm{b}}$, F. Duprat $^{\mathrm{a}}$, T. de \\ Larrard $^{\mathrm{a}}$, M. Oumouni ${ }^{\mathrm{b}}$ \\ ${ }^{a}$ LMDC, INSA/UPS Gnie Civil, 135 Avenue de Rangueil, 31077 Toulouse, cedex 04, France; \\ ${ }^{\mathrm{b}}$ Université de Nantes, Research Institute in Civil and Mechanical Engineering (GeM), UMR \\ CNRS 6183, 2 rue de la Houssinière, BP 92208, 44322 Nantes cedex 3, France
}

\section{ARTICLE HISTORY}

Compiled June 4, 2019

\begin{abstract}
Characterising spatial variability, which is of utter importance in inspection and maintenance strategies, requires comprehensive spatially distributed databases. However, in real practice, spatially distributed inspection is costly and could damage the structure if a large number of destructive tests are carried out. Therefore, the first objective of this work is to propose a methodology to extract as much informations as possible from available spatially distributed databases, in order to characterise the spatial correlation. Moreover, a preventive maintenance strategy should be supported by deterioration models able to propagate uncertainty and spatial variability. Then, the second objective of the paper is to evaluate the ability of these models to propagate uncertainties and spatial variability. The methodology is illustrated with data collected through destructive tests in a concrete wall exposed to carbonation. The database encompasses information about the concrete porosity, saturation degree, density, and carbonation depth. Recommendations are hence provided in this work for the choice of input parameters that should be modelled as random fields. These recommendations were applied and then confirmed by comparing measured and modelled spatially distributed carbonation depths. The results highlight that uncertainties in measurements and statistical uncertainties have significant impact when dealing with spatial variability.
\end{abstract}

\section{KEYWORDS}

Reinforced concrete; modelling; Carbonation; Spatial variability; Random field; Uncertainty quantification

\section{Introduction}

Corrosion of reinforcing bars has been identified among the firsts mechanisms producing a premature deterioration, lifetime reduction and therefore larger maintenance and rehabilitation costs for reinforced concrete (RC) structures. The annual cost of 5 corrosion worldwide is estimated to exceed $\$ 1.8$ trillion, which translates to $3-4 \%$ of the Gross Domestic Product (GDP) of industrialised countries (Schmitt, 2009). Since the direct and indirect costs of corrosion are immense, many studies related with preventive maintenance strategies against $\mathrm{RC}$ corrosion have been carried out for decades (Bastidas-Arteaga and Schoefs, 2012, 2015; Engelund and Sorensen, 1998; O'Connor 
et al., 2013; Tesfamariam et al., 2018). These research works highlight the importance of accounting for uncertainties in lifetime assessment and maintenance optimisation; nevertheless, their predictions could be improved by considering the spatial variability of materials and deterioration processes.

Stewart (2006) and Stewart and Mullard (2007) pointed out that accounting for spatial variability has significant impact on lifetime assessment and maintenance optimisation. As a consequence, spatial variability characterisation of concrete physical properties have been a topic of recent studies (Karimi et al., 2005; Kenshel, 2009; Li, 2004; Moshtaghin et al., 2017; Othmen et al., 2018; Schoefs et al., 2017a, 2016; Zhu et al., 2017). Such characterisation requires a large number of measurements on dif20 ferent points of the concrete surface. Spatial inspection could be performed by using accurate non-destructive techniques or sensors; however, further technical developments are necessary to obtain reliable measurements (Gomez-Cardenas et al., 2015; Schoefs et al., 2017b; Torres-Luque et al., 2014, 2017; Villain et al., 2017). In current practice, the quantity of measurements is low, due to high inspection costs and limited 25 resources allocated to maintenance policies. Therefore, increasing the possibilities of extracting as much information as possible from the collected measurements becomes a crucial challenge for spatial variability characterisation.

On the other hand, the use of predictive deterioration models is essential to optimise the resource allocation in the formulation of optimal maintenance strategies.

30 Representative models should be also able to deal with the spatial variability of model parameters, material properties or environmental exposure. This issue was recently addressed by Rakotovao Ravahatra et al. (2017) where a methodology was proposed for ranking deterioration models with respect to their capability to propagate spatial variability. The outcomes of this study provided a first attempt for establishing practi35 cal recommendations about the selection of models. However, they could be improved by studying for each model which parameters could be represented as random variables or random processes as well as considering the uncertainty in the identification of the spatial correlation parameter.

Within this context, the first objective of this paper is to provide a methodology to 40 assess the spatial correlation of model parameters or material properties. Some interesting recent studies dealt with spatial variability (Cameletti et al., 2012; , Lindgren et al.; Wang et al., 2018). However their analysis did not focus on the fact that the amount of available data could be particularly limited, as it is the case in real civil engineering applications. The methodology proposed in this paper aims at extracting as much information as possible of spatially distributed data to identify the range of variation and the mean of the parameter characterising the spatial correlation. The second objective is related to the improvement of the analysis of the capability of deterioration models according to the aspects mentioned previously. The proposed methodology is applied to a database collected during one of the experimental cam50 paigns of the ANR-EVADEOS project ${ }^{1}$. The data concerns destructive tests on a concrete wall for determining the spatial variability of inputs (porosity, saturation degree, concrete density) and output (carbonation depth) of carbonation models. The present study will focus only on concrete carbonation; nevertheless, the proposed methodology could be extended to chloride ingress or other deterioration processes and/or material 55 properties.

The paper is organised as follows. We describe in section 2 the structure investigated

\footnotetext{
${ }^{1}$ Non-destructive evaluation of the structures for damage prediction and optimisation of the follow-up. Website: http://www-lmdc.insa-toulouse.fr/evadeos/accueilevadeos.htm
} 
and data used in this work. In section 3 we provide details concerning the proposed methodology for the assessment of the range of variation and mean of the parameter characterising the spatial variability of the inputs and outputs of carbonation models. In section 4 we present the methodology and results of the sensitivity analysis aiming to evaluate the ability of carbonation models to propagate the spatial variability. This section uses simulated data to generate a significant database to propagate uncertainties and/or spatial variability on the model inputs. The methodology proposed in section 3 is also implemented in section 4 to identify the parameter characterising the 65 spatial variability. The main outcome of section 4 will be to provide recommendations about the modelling of model inputs as random variables or fields. These recommendations are tested in section 5 by utilising the real inspection data. Finally, we provide in section 6 some remarks concerning the "nugget effect" that will be considered in future works to improve the spatial variability characterisation.

\section{RC structure study case}

We investigate a RC wall built in 1979 enclosing a yard where inert wastes are stored (Rakotovao Ravahatra et al., 2017). The portion of wall studied is east-west oriented and $3.5 \mathrm{~m}$ length (Figure 1). There is no inhomogeneity due to casting or exposure conditions. It is assumed in the following that random fields are stationary and er-

75 godic; as a consequence spatial variability can be modelled using a correlation function (Schoefs et al., 2017a). 21 successive measurements were taken from cores along a single horizontal line $1.5 \mathrm{~m}$ above the ground. They were located between reinforcement meshes with a constant distance of $16 \mathrm{~cm}$ between measurements. Cores were extracted according to EN-13-791 (2007) and immediately placed into sealed plastic bags and measurements were conducted in lab. Porosity, saturation degree and concrete density were determined following the procedures described in NF-18-459 (2010) The distance of the measurement line to the ground $(1.5 \mathrm{~m})$ and to the top $(0.8 \mathrm{~m})$ was selected to avoid border effects. It was found that exposure conditions after 35 years of each wall side are rather different: on the South side, the drying is faster and carbonation is supposed to be facilitated. The mean value of carbonation depth is $1.96 \mathrm{~cm}$ for the North side (Side A) and $2.42 \mathrm{~cm}$ for the South side (Side C). It was therefore decided to consider separately the measurements obtained on each side. In Figure 2 are shown the measured values of porosity, saturation degree, concrete density and carbonation depth for the two exposed sides of the wall. The position of the measurement along the wall is located by its abscissa on the horizontal axis, while the value of the measurement of the parameter studied is given on the vertical axis. It is observed that there is a significant spatial variability and that values differ for each side.

\section{Characterisation of spatial correlation}

A trajectory is defined as a successive set of measurements along an horizontal line 95 on the surface of the structure. The set of measurements for each side in Figure 2 are examples of trajectories. Given that only one sample path (trajectory) of each random field is available for each side of the wall and for the sake of simplicity, we assume that the random fields are ergodic and Gaussian; this means that one sample (one trajectory of measurements) is sufficient to fully characterise the random field. 100 Otherwise we can carry out as previous step the pre-treatment proposed by (Clerc 
and Mallat, 2003). In addition, as well as for many study cases in civil engineering where the amount of data is insufficient (Kenshel, 2009; Li, 2004; O'Connor et al., 2013), we assume second order stationarity. This means that the mean value, the standard deviation are constant, and the autocorrelation function depends only on the distance. The objectives of this section are to describe and illustrate the proposed method called "windowing" for identifying the range of variation and the mean value of the parameter characterising the autocorrelation function. Section 3.1 provides the basis for the estimation of the parameters that characterise the spatial variability. The proposed windowing method including the criterion to determine the minimum number of points considered in the analysis are described in sections 3.2 and 3.3 , respectively. The methodology is finally illustrated in section 3.4.

\subsection{Parameters estimation}

Let $X(x)$ be a stationary Gaussian random with mean $\mu_{X}$, the variance $\sigma_{X}^{2}$ and the correlation function $\rho(\Delta x)$. The field $X$ is composed by $m$ spatially correlated positions $x_{1} \ldots, x_{m}$. Many of the correlation functions $\rho(\Delta x)$ (Table 1 ) were proposed in the literature (see for instance (Der Kiureghian and Ke, 1988; Kenshel, 2009) for an overview). They are characterised by the scale of fluctuation $\theta$. The scale of fluctuation is related to the distance from which two values from the same random field can be considered more or less dependent from another. Identifying the autocorrelation of a given physical property aims to determine the appropriate type of continuous autocorrelation function and to estimate the corresponding scale of fluctuation $\theta$.

For illustrative purposes, we consider in this work the exponential autocorrelation function, generally used for representing the spatial variability of concrete properties or durability indicators (Kenshel, 2009; Schoefs et al., 2017a, 2016): $\rho(\Delta x)=$ $\exp (-|\Delta x| / b)$ where $b=\theta / 2$. We recall that the correlation matrix $R(b)$ is defined by entries $R_{i, j}(b)=\rho\left(x_{i}-x_{j}\right)$, for $i, j=1, \ldots, m$. There are two widely used procedures for the estimation of $b$ : Maximum Likelihood Estimation (MLE) and least square (LSM) methods. The LSM is very simple and can be used even for non-Gaussian distributions, it consists in fitting an empirical covariance function $\hat{C}(\cdot)$ defined in eq. (1) with the parametric model $\sigma_{X}^{2} \rho(\cdot)$.

$$
\hat{C}(h)=\frac{1}{N_{h}} \sum_{i}\left(X\left(x_{i}\right)-\tilde{\mu}_{X}\right)\left(X\left(x_{i}+h\right)-\tilde{\mu}_{X}\right),
$$

where $\tilde{\mu}_{X}:=\frac{1}{m} \sum_{i=1}^{m} X\left(x_{i}\right)$ is the unbiased estimate of $\mu_{X}, N_{h}$ being the number of points distant with $h$ from all locations of study. The fitted parameters from eq. (1) are biased since $\hat{C}$ is a biased estimator of $\sigma_{X}^{2} \rho(\cdot)$. The MLE consists in searching for the value of $b$ that maximises the joint probability density of the data. It gives estimates with minimal variance and asymptotic normal limit. We note $\zeta:=\left(\mu_{X}, \sigma_{X}^{2}, b\right)$ and $\widehat{\zeta}:=\left(\hat{\mu}_{X}, \hat{\sigma}_{X}^{2}, \hat{b}\right)$ its estimate by MLE, this later is computed by minimising the negative log-likelihood (Clerc et al., 2019; Oumouni and Schoefs, 2019),

$$
\ell(\zeta, X)=\frac{1}{2}\left(m \log \left(\sigma_{X}^{2}\right)+\log |R(b)|+\frac{1}{\sigma_{X}^{2}}\left(X-\mu_{X}\right)^{t} R(b)^{-1}\left(X-\mu_{X}\right)\right)
$$


where $|R(b)|$ is the determinant of $R(b)$. In practice, an iterative resolution is preferred than direct optimisation procedure. This iterative procedure is summarised by the following steps:

(1) we choose an initial estimate $\hat{b}=b_{0}$, and we compute its corresponding estimate of the mean $\mu_{X}$ and variance $\sigma_{X}^{2}$, both as follows:

- MLE of the mean $\mu_{X}$ :

$$
\hat{\mu}_{X}=\frac{X^{\prime} R(\hat{b})^{-1} \mathbf{1}}{\mathbf{1}^{\prime} R(\hat{b})^{-1} \mathbf{1}}
$$

where we note by 1 the vector with $m$ entries all equal 1 .

- MLE of the variance $\sigma_{X}^{2}$ :

$$
\hat{\sigma}^{2}=\frac{\left(X-\hat{\mu}_{X}\right)^{\prime} R(\hat{b})^{-1}\left(X-\hat{\mu}_{X}\right)}{m}
$$

(2) We compute $\hat{b}$ by minimising $\ell$ knowing $\hat{\mu}_{X}$ and $\hat{\sigma}_{X}^{2}$ from step 1 .

(3) We repeat these steps until convergence.

This iterative procedure is stopped when two successive estimates $\hat{b}$ of $b$ are close to a fixed threshold.

\subsection{Windowing methodology}

The application of the MLE procedure to a single trajectory will provide only one estimate of $\hat{b}$ which remains a sample value among other possible values that could be supplied if new trajectories are available. Hence the estimated parameter $\hat{b}$ can be seen as a random variable whose uncertainty is to be determined. Taking into account that just one trajectory is available in this case and that the measures are correlated for this trajectory, the main focus of the methodology is to identify a range of variation of $\hat{b}$ as well as its mean value $\mu_{\hat{b}}$. Towards this aim, we discretise a trajectory containing $N$ spatial correlated measures into several windows where a value of $\hat{b}$ is estimated from each the trajectory trapped in the window (Figure 3). Figure 4 summarises the steps of the proposed methodology:

(1) Assessment of the minimum number of points per window $n_{w, m i n}$ : this value is determined for each trajectory following the procedure detailed in section 3.3.

(2) Window definition: we consider a window with a fixed number of successive measurements $n_{w} \in\left[n_{w, \min }, N\right]$ from which we identify a value of $\hat{b}$ using the MLE method (section 3.1).

(3) Windowing: we shift the window along the trajectory, and for each position, we identify a new value of $\hat{b}$. In Figure 3 are shown various positions of a window containing $n_{w}=4$ measurements.

(4) Increasing window size: we consider a larger window with $n_{w}+1$ measurements and we repeat the steps 2 and 3 . We increase the length of the window and we repeat the steps 2 and 3 until all measurements are considered $\left(n_{w}=N\right)$.

(5) Post-processing: the identified values of $\hat{b}$ for $n_{w} \in\left[n_{w, \min }, N\right]$ are considered to determine the range of variation (minimum and maximum value) as well as the mean of $\hat{b}$. 
where $\hat{\sigma}_{X}^{2}$ is the estimate of $\sigma^{2}$ defined in eq. (4).

Equation (6) is therefore used to determine $n_{w, \min }$ given a certain threshold $v_{t h}>0$ as follows:

$$
\delta_{\mu_{X}}:=\frac{q_{\alpha} \hat{\sigma}_{X}}{\sqrt{\mathbf{1}^{\prime} R(\hat{b})^{-1} \mathbf{1}}} \approx v_{t h}
$$

The minimum number of measurements $n_{w, \min }$ for which the indicator $\delta_{\mu_{X}}$ satisfies eq. (7) ensures also that the mean $\mu_{X}$ respects the following accuracy:

$$
\mathbb{P}\left(\hat{\mu}_{X} \in\left[\mu_{X}-v_{t h}, \mu_{X}+v_{t h}\right]\right) \approx 1-\alpha
$$

\subsection{Application of the windowing method to the study case}

proposed methodology is illustrated in this section considering the data measured in the side A for the carbonation depth. Following the procedures given in sections 3.2 and 3.3, Figure 5 provides the variance of the mean estimate $\operatorname{var}\left[\hat{\mu}_{X}\right]$ for $\alpha=10 \%(90 \%$ confidence interval) and various values of $n_{w}$. It is observed that $\operatorname{var}\left[\hat{\mu}_{X}\right]$ decreases up to a minimum value of 0.5 when $n_{w}=N$. For the other trajectories, the minimum $\operatorname{var}\left[\hat{\mu}_{X}\right]$ varies between 0.4 and 0.5 . Taking into account these findings, we selected a threshold value $v_{t h}=0.6$ to determine $n_{w, \min }$. Table 2 gives the values of $n_{w, \min }$ computed for all the parameters. We found different values ranging from 8 to 15 for each parameter and side. Since our database is limited to generalise these findings, we 
suggest to assess and use a given $n_{w \text {,min }}$ per trajectory. The values given in Table 2 will be used in this paper.

When carrying out the previous numerical procedure, we obtained for each length of window and each position, a value of $\hat{b}$. In Table 3 , we provide an example of the assessment of $\hat{b}$ with the windowing method and measurements of porosity. We observe that when $n_{w}$ varies between 4 and 11, the identification process does not converge (NC) for some positions of the window. The non-convergence of the MLE method occurs often for windows with fewer number of measurements. Moreover, results in (Rakotovao Ravahatra et al., 2017) showed that for $n_{w}<10$, statistical uncertainties in the computation of discrete spatial autocorrelation are significant. For this case we retain the values in green coloured cells that satisfy the condition $n_{w} \in\left[n_{w, \min }, N\right]$.

In Figure 6 are depicted the results of identification of $\hat{b}$ for all measured parameters and for the two sides (A and C) of the wall. More or less significant differences appear on the mean values of $b$ between the two sides. This can be due to both the effect of degradation and the exposure conditions. Particularly, one observes in Figure 2 that the mean value of porosity and saturation degree are lower and carbonation depth is larger for the side C. This side is south exposed and then prone to exhibit a faster drying of the surface of the concrete, favourable to carbonation. Therefore the data observed are consistent with the process of the degradation. No significant difference is observed for concrete density.

Besides it can be noted in Figure 6 that the values of the mean of $\hat{b}$ for the concrete density are close on both sides indicating that both faces of the wall are made of the same initial material (the concrete was coming from the same batch when poured and with the same process of concrete vibration). Then it is possible to suggest that the impact of carbonation or exposure conditions on the spatial correlation of concrete density is negligible. The mean values of $\hat{b}$ for the porosity and carbonation depth are more important on side $\mathrm{A}$ than on side $\mathrm{C}$. This can be attributed to the effect of the process of carbonation that is more pronounced on side $\mathrm{C}$ and brings additional scatter on the physical properties of the material. This leads hence to less spatial correlation of material properties that are modified by carbonation process (porosity and carbonation depth). The mean of $\hat{b}$ for the saturation degree is more important on side $\mathrm{C}$ than on side $\mathrm{A}$. As this property mainly depends on exposure conditions, a variation between both sides is expected.

According to the previous observations, the spatial autocorrelation of the concrete physical properties that are influenced by carbonation process (porosity and carbonation depth) depends on the current deterioration state driven by carbonation. Given these results, we could reasonably conclude that the spatial autocorrelation of porosity and carbonation depth changes over time, -i.e. $\hat{b}$ is a function of time for these parameters. In order to confirm these findings, data collected at several other time steps would be required.

\section{Sensitivity of concrete carbonation models to input random fields}

245 An optimal maintenance strategy should be supported by models able to predict the corrosion onset caused by concrete carbonation. Given that concrete properties as well as carbonation depth are spatially variable, it is necessary to analyse the ability of models to transfer the spatial correlation of input parameters. We should also assess the influence of each input parameter when it is modelled as a random field. This study extends a previous work (Rakotovao Ravahatra et al., 2017) where the uncertainty on 
the assessment of $b$ was not investigated. Since previous results (section 3.4) showed that this uncertainty is significant, we carry out a sensitivity analysis on concrete carbonation models by considering model inputs as independent random fields and varying the value of $b$. The carbonation models studied in this paper are those which require the physical parameters that are usually investigated in existing structures (porosity, saturation degree, concrete density): Hyvert (2009), Papadakis et al. (1991), Miragliotta (2000) and Ying-Yu and Qui-Dong (1987). According to the review of Rakotovao Ravahatra et al. (2019), we provide a summarised description of these models in Appendix A.

\subsection{Methodology}

In the first part of the study, we will focus on evaluating separately the influence of considering each model input as a random field. Towards this aim, whereas one input is modelled as a random field, the other inputs are set as deterministic values (mean values). Afterwards, we will consider that all the inputs are represented by random fields (reference case). We summarise in Table 4 the cases studied. The model parameters not mentioned in Table 4 are represented as deterministic values and are provided in Table A3.

The objective being to adopt a wide view and to be able to generalise the results; the sensitivity analysis will consider several values of the autocorrelation parameter $(b=5 \mathrm{~cm}, 50 \mathrm{~cm}$ or $100 \mathrm{~cm})$ for the input parameters modelled as random fields. 5 $\mathrm{cm}$ appears to be an acceptable minimum with respect to the size of aggregates, 50 $\mathrm{cm}$ is close to the distance between two successive points of vibration when pouring concrete, and $100 \mathrm{~cm}$ corresponds to the maximum value of $b$ found in the literature (Kenshel, 2009).

The sensitivity analysis concerns the following steps:

(1) to generate 100 trajectories (sample paths) for each value of $b(5 \mathrm{~cm}, 50 \mathrm{~cm}$ and $100 \mathrm{~cm}$ ) using the Karhunen-Loève expansion (Karhunen, 1947; Loeve, 1948);

(2) to compute the model output (spatial distribution of the carbonation depth at 35 years of exposure) corresponding to the generated input trajectories for the considered carbonation models (Appendix A);

(3) to compute the simulated discrete autocorrelation function of the output and to identify the corresponding values of $\hat{b}$ for the 100 trajectories of carbonation depth using the windowing method described in section 3 ;

(4) to analyse the effects of the spatial correlation of inputs on the spatial variability of the output (carbonation depth assessed from models).

\subsection{Results}

We present the results in Figures 7, 8, 9 and 10. In these figures are reported results of simulated and empirical discrete autocorrelation, and the histograms of the identified $\hat{b}$ from the output of the models. On simulated discrete autocorrelation curves, point marks represent the mean values and dotted lines provide the $10 \%$ and $90 \%$ quantiles values over 100 simulated discrete autocorrelation functions at each $\Delta x$. On the empirical discrete autocorrelation function, points describe the autocorrelation values obtained from measurements and dotted lines provide the bounds determined when uncertainties in measurements and statistical uncertainties are taken into account (Rakotovao Ravahatra et al., 2017). Concerning the histograms of $\hat{b}$, we present 
on each figure the results corresponding to a given value of $b(5 \mathrm{~cm}, 50 \mathrm{~cm}$ and 100 $\mathrm{cm}$ ) for the input considered. In the following sections, we analyse the results for the cases described in Table 4.

\subsubsection{Sensitivity to the porosity random field}

We report in Figure 7 the results for each carbonation model when the porosity is modelled as a random field (Case 1 in Table 4).

Regarding simulated discrete autocorrelation functions, the results for all models are similar. When $b$ increases, the gap between minimum and maximum values (scatter) is smaller. One observes hence in the spatial correlation of the models outputs the same tendencies as for the spatial correlation of the porosity. Indeed, when we increase the value of $b$ for the porosity, the realisations of the porosity random field are more correlated and less uncertain. This indicates that the correlation of the porosity is quite well transferred to the model output (simulated carbonation depth).

The ratio of the mean value of $\hat{b}, \hat{\mu}_{b}$, with respect to the value of $b$ (i.e., $\hat{\mu}_{b} / b$ ) for 10 a given input provides a quantification of the ability of the model to transfer spatial correlation from the inputs to the output. We present in Table 5 the ratios $\hat{\mu}_{b} / b$ for each input parameter, each case, and each model.

Regarding porosity (case 1 ), we can observe that for all models there is an amplification $\left(\hat{\mu}_{b} / b>1\right)$ of the spatial correlation when $b \leq 50 \mathrm{~cm}$. On the contrary there is a reduction of the spatial dependency when $b=100 \mathrm{~cm}$. The uncertainties in measurements and statistical uncertainties (Rakotovao Ravahatra et al., 2017) could explain these results. Indeed, we can see in Figure 7 (black coloured curves) that these uncertainties are quite significant and could have important influence in the identification of $\hat{b}$. The presence of uncertainty brings additional difficulties to the analysis and characterisation of the spatial correlation.

\subsubsection{Sensitivity to the saturation degree random field}

We provide in Figure 8 the results for each carbonation model when saturation degree is modelled as a random field (Case 2 in Table 4).

Concerning simulated discrete autocorrelation functions, we can observe significant differences between models. It is difficult to establish a constant value of $b$ over time for the saturation degree because this parameter highly depends on the exposure conditions during the inspection. Nevertheless it can be stated that the mean value of $b$ for this parameter could lie between $5 \mathrm{~cm}$ and $50 \mathrm{~cm}$ when comparing simulated and empirical discrete autocorrelation functions. This confirms the results in Figure 6.

330 Regarding the model of Ying-Yu, modifying the value of $b$ has no significant impact. Especially, it is observed that between $b=50 \mathrm{~cm}$ and $100 \mathrm{~cm}$ the results are very similar. On the other hand, it is noted that the autocorrelation of the model output is quite low whatever the value of $b$ for the saturation degree. These findings indicate that high correlations of the saturation degree are not transferred to the Ying-Yu model output. The scatter of the simulated discrete autocorrelation is slightly higher for the models of Hyvert and Papadakis and larger than when the porosity is considered as a random filed. These models appear to be more sensitive to saturation random field. We can observe similar trend as for porosity for the model of Miragliotta.

Regarding the histogram of $\hat{b}$, we can confirm the same findings observed for the simulated discrete autocorrelation functions. After propagation in the models, the spatial variability of the saturation degree is less amplified and slightly more reduced 
than the one imposed by the porosity. Indeed, we can observe in Table 5 that the ratios $\hat{\mu}_{b} / b$ for the Case 2 are lower than the Case 1 . Concerning the ranges of variation, they are slightly higher than for Case 1 excepting for the model of Ying-Yu for which it is drastically amplified for $b=50 \mathrm{~cm}$ and $b=100 \mathrm{~cm}$. These results indicate that excepting the model of Ying-Yu, all the models studied well transfer lower and higher spatial correlation of saturation degree, and are hence sensitive to spatial correlation of this input parameter.

\subsubsection{Sensitivity to the concrete density random field}

350 In Figure 9 are reported the results obtained when the concrete density is considered as a random field (Case 3 in Table 4). The results concern only the model of Ying-Yu because it is the only one that uses the concrete density as an input parameter.

Regarding simulated discrete autocorrelation, in comparison with the previous results, one observes that the scatter is larger for $b=5 \mathrm{~cm}$. For $b=50$ and $100 \mathrm{~cm}$ there is an effect on the mean value of the autocorrelation but the scatter is negligible. These findings indicate that Ying-Yu model is highly sensitive to the choice of the value of $b$ used to represent the spatial variability of concrete density.

Concerning the histogram of $\hat{b}$, even if there is no scatter in the simulated discrete autocorrelation function when the imposed value of $b$ is equal to $50 \mathrm{~cm}$ and $100 \mathrm{~cm}$ for density, it is observed an important scatter in the estimated $\hat{b}$. On the other hand, the spatial dependency significantly raises after being transferred through the model. Indeed, $\hat{\mu}_{b}$ for the simulated carbonation depth is 4 times higher than $b$ when this latter is equal to $5 \mathrm{~cm}$ (Table 5 ). However, $\hat{\mu}_{b} / b<1$ when $b=100 \mathrm{~cm}$. The same trend was observed in the previous cases.

\subsubsection{Random fields of all inputs}

In Figure 10 are depicted the results obtained when all the parameters (porosity, saturation degree and density) are modelled as random fields (Case 4 in Table 4).

Concerning simulated discrete autocorrelations, first, we can observe that scatter is wider for the models of Hyvert and Papadakis, while it is smaller for the model 370 of Ying-Yu. The model of Miragliotta is an intermediate between the 2 tendencies. This indicates that some models (Hyvert and Papadakis) are more sensitive to input random fields when compared to others. Second, it is noted that the scatters estimated in Figure 10 are quite similar to those of Figure 8 (random field for saturation degree) for the models of Hyvert, Papadakis and Ying-Yu. This indicates that the spatial variability of the output is highly influenced by the spatial variability of saturation degree for these models. Regarding the Miragliotta model, the scatters when varying $b$ for saturation degree (Figure 8) are wider than when varying it for porosity (Figure 7 ). However, when varying $b$ for all inputs, the scatters appear to be similar to results in Figure 7 indicating mofe sensitivity to the random field of porosity. This indicates that the Miragliotta model is more sensitive to the spatial variability of the porosity random field. Finally, whatever the correlation of any input of Ying-Yu model, the outputs would always be uncorrelated. Therefore, describing spatial variability with this model appears to be difficult.

Concerning the histogram of $\hat{b}$, one observes the same tendencies as with simulated discrete autocorrelation function. Indeed, the histograms are similar to Figure 8 for all models except for the model of Miragliotta which presents the same tendencies as in Figure 7. 
We present in Table 6 the Absolute Error (AE) on $\hat{\mu}_{b}$ for the Cases 1 to 3. This AE is estimated taking as a reference the $\hat{\mu}_{b}$ of Case 4 . The results show that the lowest AE are for the Case 2 and for the models of Hyvert, Papadakis and Ying-yu. Therefore, the saturation degree appears to be the most influential parameter for these models with respect to spatial correlation propagation. Regarding the model of Miragliotta, the lowest error corresponds to the Case 1 . The spatial variability of the output of this model is hence strongly influenced by the spatial variability of the porosity.

Concerning the model of Ying-Yu, the Case 2 leads to similar results than Case 4 (see Table 6). This implies that choosing this case will provide simulated carbonation depth with lower spatial correlation. Therefore, the AE analysis is not useful for the Case 2. Following the findings found in Table 5, the Case 1 appears to be better for propagating spatial variability in this model.

The findings of this sensitivity analysis were used to propose the recommendations summarised in Table 7. For instance, it was found that the saturation degree is the most influencing random field for the models of Hyvert and Papadakis. The model of Miragliotta is driven by the spatial variability of the porosity, and the model of Ying$\mathrm{Yu}$ can only deal with the spatial variability of the porosity. The results of this table can be used to define which parameters should be prioritised in inspection campaigns when the objective is to characterise and propagate spatial variability.

\section{Evaluation of the recommendations of the sensitivity analysis}

In this section we test the previous recommendations (Table 7) to analyse how efficient they are when dealing with the real database obtained during the inspection work presented in Section 2. The following steps are proposed for this study:

(1) Define the study cases according to the recommendations given in Table 7. Table 8 provides a description of these study cases. In the full case all the parameters are modelled as random fields. The "Reduced 1" and "Reduced 2" cases follow respectively the findings of first (R1) or second (R2) priorities given in Table 7 to determine if the parameters should be modelled as random field or constant values. The constant value is equal to the mean determined from the data reported in Figure 2.

(2) Propagate the spatially correlated data reported in Figure 2 into the carbonation models for each case.

(3) Use the windowing method presented in section 3 to estimate the variability of $\hat{b}$ for each model and case.

(4) Evaluate the effectiveness of the recommendations by comparing the histograms obtained after propagating spatial variability for each model and case with those estimated from the measurements of carbonation depth (Figure 6). The comparison is carried out in terms of the AE between the $\hat{\mu}_{b}$ for each model and the $\hat{\mu}_{b}$ for measurements (Table 9).

\subsection{Full}

We present results for all models and for the 2 sides of the wall in the Figure 11. On the side A, we can observe that the models of Hyvert and Ying-Yu appear to underestimate the correlation and provide hence slightly lower values of $\hat{b}$. Regarding the models of Papadakis and Miragliotta the histograms of $\hat{b}$ overlap those identified 
from measurements. However, no significant differences could be observed between models with respect to side $\mathrm{A}$. On this side, we can also note that the values of $\hat{b}$ for porosity are low when they are slightly higher for saturation degree (see Figure 6). Excepting the model of Ying-Yu, results of the previous sensitivity analysis show that all models are sensitive to the spatial variability of porosity and saturation degree (see Figures 7 and 8). The effect of slightly higher correlation of porosity appears to be compensated by lower correlation of saturation degree. Consequently we observe the intermediate tendencies on the Figure 11.

440 Regarding the side $\mathrm{C}$, none of the histograms of $\hat{b}$ obtained using the models overlaps the one from measurements and differences between models are more important. One observes that the models of Hyvert and Papadakis slightly overestimate $\hat{b}$, while the models of Miragliotta and Ying-Yu underestimate it. On the contrary for side A, the identified values of $\hat{b}$ are lower for porosity in this side, while they are higher for 445 saturation degree (see Figure 6). It appears through these results that the models of Hyvert and Papadakis are more sensitive to higher correlation of saturation degree while the models of Miragliotta and Ying-Yu are more sensitive to lower correlation of porosity. On the other hand, concerning the last two models (Miragliotta and Ying$\mathrm{Yu}$ ), the interval of values of $\hat{b}$ is smaller when compared to the measurements, -i.e.

450 the dispersion is reduced. This is in agreement with the results in Figure 10 where we can see that for these models the transfer of dispersion is lower when compared to the models of Hyvert and Papadakis.

\subsection{Reduced 1}

Regarding the models of Hyvert and Papadakis, and for the two sides of the wall, the $\hat{\mu}_{b}$ is close to the results reported in Figure 11. Concerning the models of Miragliotta and Ying-Yu and for the side A, the values of $\hat{\mu}_{b}$ in Figure 11 are higher than those of Figure 12. For the side $\mathrm{C}$, the values of $\hat{\mu}_{b}$ are similar to the found for the Full cases for all the models. In Table 9, it is noted that when considering the Reduced 1 cases, the $\mathrm{AE}$ on the assessment of $\hat{\mu}_{b}$ are close to the values obtained for the Full case. These results found when only one random field is propagated in the models may indicate that the recommendations in Table 8 could be sufficient to represent the spatial variability of the problem.

\subsection{Reduced 2}

Concerning the models of Hyvert and Papadakis and for the side A, one observes that 465 the histograms of $\hat{b}$ of the model outputs overlap that of the measurements. However, the ranges of variation are widely higher when $\hat{b}$ is estimated after propagating spatial variability in such models. Regarding the models of Hyvert and Papadakis for the side $\mathrm{C}$ and the models of Miragliotta and Ying-Yu for both sides, one observes that the number of identified values of $b$ is small. This is due to a large number of non-

470 convergences found during the simulations. On the other hand, for the model of Ying$\mathrm{Yu}$ and side A, any value of $\hat{b}$ was identified for the case Reduced 1 (bis). Then, we present in Figure 14 only the results for the side B.

It is noted in Table 9 that the $\mathrm{AE}$ are smaller in comparison with the other cases. However, it is not possible to provide a recommendation based on this indicator because: (i) the assessment of $\hat{\mu}_{b}$ is based in few data (due to the large number of non-convergences), and (ii) there are significant differences between the maximum 
and minimum bounds when comparing the histograms obtained from measurements and models.

\subsection{Summary of recommendations}

480 Previous results highlight that recommendations in Table 7 could be convenient for all models, especially for the models of Hyvert and Papadakis. However, these recommendations are less efficient for the models of Miragliotta and Ying-Yu with respect to the side C. This inefficiency could be related to uncertainties in measurements which were more important in side $\mathrm{C}$. The increase of the AE for $\hat{\mu}_{b}$ for all models may indi-

485 cate non negligible correlation between the models inputs, especially for the models of Miragliotta and Ying-Yu. However, in order to confirm this assumption, further data is required.

\section{Nugget effects}

When analysing auto-covariance, we can observe an abrupt change of the value of the covariance in the beginning of the curve $(\Delta x=0)$. This discontinuity is called "nugget effect" in the field of geostatistics. This may bring additional uncertainty when modelling spatial variability. The nugget effect in variogram analysis was dealt with in some studies (Wagner et al., 2005). Its effect in kriging was also handled by Brooker (1986) or Yin et al. (2011). Concerning the autocorrelation function, we should carry out a similar analysis to the reported in the above-mentioned studies when we observe nuggets in the discrete autocorrelation function. Further work will focus on the development of a methodology to take into account nugget effects in spatial variability assessment.

\section{Conclusions and perspectives}

The following conclusions are drawn from the present study:

- The proposed windowing method is useful for spatial variability characterisation when the spatially correlated data is scarce. It allow us to determine the range of variation and the mean of the parameter used to model the spatial correlation.

- Significant differences for the range of variation and mean value of $\hat{b}$ were found for each side of the wall for the porosity and carbonation depth. Since carbonation modifies along time concrete porosity, saturation degree and concrete density, we can suppose that the autocorrelations of these physical properties will also become time-dependent. Further data collected at several time steps is required to confirm this assumption.

- A sensitivity analysis aiming to test the ability of concrete carbonation models to propagate spatial variability was useful to provide recommendations about the input parameters that could be modelled as random fields. This sensitivity analysis was based on simulated data by considering several values of the autocorrelation parameter $b$.

- The recommendations were tested with the real and scarce database of the case study. This analysis highlighted that the recommendations are rather convenient for all models, especially for the models of Hyvert and Papadakis. 
- The proposed methodology and findings of this paper could help to decisionmakers to define which parameters are more appropriate to measure and which models are more accurate to use when dealing with spatially deteriorated practical applications. This methodology could be also applied to other deterioration problems.

Further work in this area will focus on:

- Obtaining a more rich spatially correlated database to test the methodology and confirm the findings and assumptions.

- Considering correlations between random fields.

- Determining which is the best type of autocorrelation function for each random field.

- Defining which variables should be modelled as random variables.

- Adapting the methodology to account for the nugget effect.

\section{Acknowledgments}

This work was made possible thanks to the French Research Agency ANR "Building and Sustainable Cities", which funded the research project ANR-EVADEOS. We are grateful to CEA-Saclay for allowing its installations to be used. Partners of the ANR-EVADEOS project are warmly thanked for the data that have been acquired and shared out (CEA Saclay, IFSTTAR Nantes, LMA Univ. Aix-en-Provence, I2M Univ. Bordeaux, EDF Chatou, LMDC Univ. Toulouse, GeM Univ. Nantes). We also acknowledge the useful comments of the anonymous reviewers to improve the final version of the paper

\section{References}

Bastidas-Arteaga, E. and Schoefs, F. (2012). "Stochastic improvement of inspection and maintenance of corroding reinforced concrete structures placed in unsaturated environments." Engineering Structures, 41, 50-62.

Bastidas-Arteaga, E. and Schoefs, F. (2015). "Sustainable maintenance and repair of RC coastal structures." Proceedings of the Institution of Civil Engineers - Maritime Engineering, 168(4), 162-173.

Brooker, P. I. (1986). "A parametric study of robustness of kriging variance as a function of range and relative nugget effect for a spherical semivariogram." Mathematical Geology, 18(5), 477-488.

Cameletti, M., Lindgren, F., Simpson, D., and Rue, H. (2012). "Spatio-temporal modeling of particulate matter concentration through the spde approach." AStA Advances in Statistical Analysis, 97(2), 109-131.

Clerc, M. and Mallat, S. (2003). "Estimating deformations of stationary processes." The Annals of Statistics, 31(6), 1772-1821.

Clerc, R., Oumouni, M., and Schoefs, F. (2019). "Scap-1d: A spatial correlation assessment procedure from unidimensional discrete data." Reliab Eng Syst Saf (under revision).

Der Kiureghian, A. and Ke, J. (1988). "The stochastic finite element method in structural reliability." Prob. Eng. Mech, 3(2), 83-91.

EN-13-791 (2007). Assessment of in-situ compressive strength in structures and pre-cast concrete components.

Engelund, S. and Sorensen, J. D. (1998). "Maintenance planning for chloride initiated cor- 
rosion in concrete structures." Structural Safety and Reliability : Proceedings of the 7th international conference on structural safety and reliability, 435-442.

Gomes, H. M. and Awruch, A. M. (2002). "Reliability of reinforced concrete structures using stochastic finite elements.." Engineering Computations, 19(7-8), 764-786.

Gomez-Cardenas, C., Sbarta, M., Garnier, V., and Balayssac, J. (2015). "NDT inspection strategy to minimize the number of samples for onsite concrete evaluation." Proceedings of Int. Symp. on NDT-CE, Berlin, Germany (15-17 September).

Hyvert, N. (2009). "Application de l'approche probabiliste de la durabilité des produits préfabriqués en béton." PhD thesis, Université de Toulouse III-Paul Sabatier, Université de Toulouse III-Paul Sabatier.

Karhunen, K. (1947). "Uber lineare methoden in der wahrscheinlichkeitsrechnung." Amer. Acad. Sci, 37, 3-79.

Karimi, A., Ramachandran, K., and Buenfeld, N. R. (2005). "Probabilistic analysis of reinforcement corrosion with spatial variability." 9th International Conference on Structural Safety and Reliability, ICOSSAR'05, Millpress, Rotterdam.

Kenshel, O. M. (2009). "Iinfluence of spatial variability ofn whole life management of reinforced concrete bridges." Ph.D. thesis, University of Dublin, Trinity college, University of Dublin, Trinity college.

Kim, H. (2005). "Spatial variability in soils: Stiffness and strength." Ph.D. thesis, Georgia Institute of Technology, Georgia.

Li, C. Q. (2004). "Reliability based service life prediction of corrosion affected concrete structures.." Ph.D. thesis, Delft University, Netherlands.

Lindgren, F., Rue, H., and Lindstrm, J. "An explicit link between gaussian fields and gaussian markov random fields: the stochastic partial differential equation approach." Journal of the Royal Statistical Society: Series B (Statistical Methodology), 73(4), 423-498.

Loeve, M. (1948). Fonctions aléatoires du second ordre. Gautiers Villars, Paris Supplement to P. Levy, "Processus stochastic et mouvement Brownien".

Miragliotta, R. (2000). "Modélisation des processus physico-chimiques de la carbonatation des bétons préfabriqués - prise en comptes des effets de paroi." Ph.D. thesis, Université de la Rochelle, Université de la Rochelle.

Moshtaghin, A. F., Franke, S., Keller, T., and Vassilopoulos, A. P. (2017). "Experimental characterization of longitudinal mechanical properties of clear timber: Random spatial variability and size effects." Construction and Building Materials, 120, 432-441 https://doi.org/10.1016/j.conbuildmat.2016.05.109.

NF-18-459 (2010). Concrete - Testing hardened concrete - Testing porosity and density.

O'Connor, A., Sheils, E., Breysse, D., and Schoefs, F. (2013). "Markovian bridge maintenance planning incorporating corrosion initiation and non-linear deterioration." ASCE journal of bridge engineering, 3, 189-199.

Othmen, I., Bonnet, S., and Schoefs, F. (2018). "Statistical investigation of different analysis methods for chloride profiles within a real structure in a marine environment." Ocean Engineering, 157(1), 96-107.

Oumouni, M. and Schoefs, F. (2019). "An adaptive approach for spatial variability assessment of structures from distributed measurements." Reliab Eng Syst Saf (under review).

Papadakis, V., Vayenas, C., and Fardis, M. (1991). "Fundamental modelling and experimental investigation of concrete carbonation." ACI Materials Journal, 4(88), 363-373.

Rakotovao Ravahatra, N., Bastidas-Arteaga, E., Schoefs, F., de Larrard, T., and Duprat, F. (2019). "Probabilistic and sensitivity analysis of analytical models of corrosion onset for reinforced concrete structures." European Journal of Environmental and Civil Engineering https://doi.org/10.1080/19648189.2019.1591307.

Rakotovao Ravahatra, N., Schoefs, F., Duprat, F., de Larrard, T., and Bastidas-Arteaga, E. (2017). "Assessing the capability of analytical carbonation models to propagate uncertainties and spatial variability of reinforced concrete structures." Frontiers in Built Environment: Bridge Engineering, 3(1), 1-9.

615 Schmitt, G. (2009). Global needs for knowledge dissemination, research, and development in 
materials deterioration and corrosion control. The World Corrosion Organization, New York, NY.

Schoefs, F., Bastidas-Arteaga, E., and Tran, T. (2017a). "Optimal embedded sensor placement for spatial variability assessment of stationary random fields." Engineering Structures, 17, $35-44$.

Schoefs, F., Bastidas-Arteaga, E., Tran, T., Villain, G., and Derobert, X. (2016). "Characterization of random fields from NDT measurements: A two stages procedure." Engineering structures, 111, 312-322 https://doi.org/10.1016/j.engstruct.2015.11.041.

Schoefs, F., Oumouni, M., Clerc, R., Othmen, I., and Bonnet, S. (2017b). "Statistical analysis and probabilistic modeling of chloride ingress spatial variability in concrete coastal infrastructures." EDITION 4, SPLIT, Croatia, Coastal and Maritime Mediterranean Conference.

Stewart, M. (2006). "Spatial variability of damage and expected maintenance costs for deteriorating rc structures." Structure and Infrastructure Engineering: Maintenance, Management, Life-Cycle Design and Performance, 2(2), 70-90.

630 Stewart, M. and Mullard, J. (2007). "Spatial time-dependent reliability analysis of corrosion damage and the timing of first repair for RC structures." Engineering structures, 29, 14571464.

Tesfamariam, S., Bastidas-Arteaga, E., and Lounis, Z. (2018). "Seismic Retrofit Screening of Existing Highway Bridges With Consideration of Chloride-Induced Deterioration: A Bayesian Belief Network Model." Frontiers in Built Environment, 4, 1-11.

Torres-Luque, M., Bastidas-Arteaga, E., Schoefs, F., Sanchez-Silva, M., and Osma, J. (2014). "Non-destructive methods for measuring chloride ingress into concrete: Stateof-the-art and future challenges." Construction and Building Materials, 68, 68-81 https://doi.org/10.1016/j.conbuildmat.2014.06.009.

640 Torres-Luque, M., Osma, J., Sanchez-Silva, M., Bastidas-Arteaga, E., and Schoefs, F. (2017). "Chlordetect: Commercial calcium aluminate based conductimetric sensor for chloride presence detection." Sensors, 17, 1-19.

Vanmarcke, E.-H. and Grigoriu, M. (1983). "Stochastic finite element analysis of simple beams." J. Eng. Mech., 109(5), 1203-1214.

645 Villain, G., Balayssac, J., and Garnier, V. (2017). Non-destructive Testing and Evaluation of Civil Engineering Structures. ISTE Press - Elsevier, 1st edition, Chapter 9, 1st edition.

Wagner, H. H., Holderegger, R., Werth, S., Gugerli, F., Hoebee, S. E., and Scheidegger, C. (2005). "Variogram analysis of the spatial genetic structure of continuous populations using multilocus microsatellite data." Genetics, 169(3), 1739?1752 doi: 10.1534/genetics.104.036038.

Wang, H., Wang, X., Wellmann, J. F., and Liang, R. Y. (2018). "Bayesian stochastic soil modeling framework using gaussian markov random fields." ASCE-ASME Journal of Risk and Uncertainty in Engineering Systems, Part A: Civil Engineering, 4(2), 04018014.

Yin, J., Ng, S., and Ng, K. (2011). "Kriging metamodel with modified nugget-effect: The heteroscedastic variance case." Computers \& Industrial Engineering, 61, 760-777.

Ying-Yu, L. and Qui-Dong, W. (1987). "The mechanism of carbonation of mortars and the dependence of carbonation on pore structure." ACI-SP 100, Concrete Durability, 1915-1943.

Zhu, F., Zhou, Q., Wanga, F., and Yang, X. (2017). "Spatial variability and sensitivity analysis on the compressive strength of hollow concrete block masonry wallettes." Construction and Building Materials, 140, 129-138 https://doi.org/10.1016/j.conbuildmat.2017.02.099. 
Table 1. Autocorrelation function $(\mathrm{ACF})$ and scale of fluctuations

\begin{tabular}{|c|c|c|c|c|}
\hline & ACF Name & $\rho(\Delta x)$ & $\theta$ & Reference \\
\hline 1 & Triangular & $\left\{\begin{array}{c}1-\frac{|\Delta x|}{a}|\Delta x| \leq a \\
0\end{array}\right.$ & $a$ & Vanmarcke and Grigoriu (1983) \\
\hline 2 & Exponential & $\exp \left(-\frac{|\Delta x|}{b}\right)$ & $2 b$ & Vanmarcke and Grigoriu (1983) \\
\hline 3 & $\begin{array}{c}\text { Second-order } \\
\text { autoregressive }\end{array}$ & $\left(1+\frac{|\Delta x|}{c}\right) \exp \left(-\frac{|\Delta x|}{c}\right)$ & $4 c$ & Vanmarcke and Grigoriu (1983) \\
\hline 4 & Gaussian & $\exp \left[-\left(\frac{|\Delta x|}{l}\right)^{2}\right]$ & $\sqrt{\pi} l$ & Vanmarcke and Grigoriu (1983) \\
\hline 5 & $\begin{array}{c}\text { Cosine } \\
\text { exponential }\end{array}$ & $\exp \left(-\frac{|\Delta x|}{e}\right) \cos \left(\frac{|\Delta x|}{e}\right)$ & $e$ & Kim (2005) \\
\hline 6 & Sinusoidal & $\frac{\sin \left[-2,2 \frac{|\Delta x|}{f}\right]}{-2,2 \frac{|\Delta x|}{f}}$ & $f$ & Gomes and Awruch (2002) \\
\hline
\end{tabular}

Table 2. Values of $n_{w, \min }$ for the studied parameters

\begin{tabular}{|c|c|c|}
\hline Parameter & $n_{w, \min }$ for Side A & $n_{w, \min }$ for Side B \\
\hline Porosity & 15 & 8 \\
\hline Saturation & 9 & 10 \\
\hline Density & 8 & 12 \\
\hline Carbonation depth & 15 & 9 \\
\hline
\end{tabular}


Table 3. Identified values of $b(\mathrm{~cm})$ for porosity (Side A), each $n_{w}$ and each position of the window

\begin{tabular}{|c|c|c|c|c|c|c|c|c|c|c|c|c|c|c|c|c|c|}
\hline \multirow{2}{*}{$n_{w}$} & \multicolumn{10}{|c|}{ Position of the corresponding window } \\
\cline { 2 - 7 } & $\mathbf{1}$ & $\mathbf{2}$ & $\mathbf{3}$ & $\mathbf{4}$ & $\mathbf{5}$ & $\mathbf{6}$ & $\mathbf{7}$ & $\mathbf{8}$ & $\mathbf{9}$ & $\mathbf{1 0}$ & $\mathbf{1 1}$ & $\mathbf{1 2}$ & $\mathbf{1 3}$ & $\mathbf{1 4}$ & $\mathbf{1 5}$ & $\mathbf{1 6}$ & $\mathbf{1 7}$ \\
\hline 4 & 8 & 16 & 7 & 8 & 18 & 12 & NC & NC & NC & NC & NC & NC & NC & 12 & NC & 12 & 16 \\
\hline 5 & 24 & 11 & 9 & 13 & 17 & NC & NC & NC & NC & NC & NC & NC & NC & NC & 11 & 19 & - \\
\hline 6 & 20 & 10 & 14 & 13 & 11 & NC & NC & NC & NC & NC & NC & NC & NC & 11 & 17 & - & - \\
\hline 7 & 13 & 14 & 13 & 10 & 11 & NC & NC & NC & NC & NC & 6 & NC & 9 & 16 & - & - & - \\
\hline 8 & 16 & 13 & 10 & 10 & 8 & NC & NC & NC & NC & NC & NC & 7 & 16 & - & - & - & - \\
\hline 9 & 15 & 10 & 10 & 8 & 7 & NC & NC & NC & NC & NC & 8 & 13 & - & - & - & - & - \\
\hline 10 & 10 & 10 & 8 & 7 & 7 & NC & NC & NC & NC & 6 & 14 & - & - & - & - & - & - \\
\hline 11 & 10 & 8 & 7 & 7 & 8 & NC & NC & NC & 6 & 12 & - & - & - & - & - & - & - \\
\hline 12 & 8 & 7 & 7 & 8 & 10 & 6 & 6 & 6 & 12 & - & - & - & - & - & - & - & - \\
\hline 13 & 7 & 7 & 8 & 10 & 11 & 10 & 6 & 12 & - & - & - & - & - & - & - & - & - \\
\hline 14 & 7 & 8 & 10 & 11 & 7 & 7 & 11 & - & - & - & - & - & - & - & - & - & - \\
\hline 15 & 8 & 10 & 11 & 8 & 10 & 12 & - & - & - & - & - & - & - & - & - & - & - \\
\hline 16 & 10 & 11 & 8 & 10 & 13 & - & - & - & - & - & - & - & - & - & - & - & - \\
\hline 17 & 11 & 8 & 10 & 13 & - & - & - & - & - & - & - & - & - & - & - & - & - \\
\hline 18 & 8 & 10 & 13 & - & - & - & - & - & - & - & - & - & - & - & - & - & - \\
\hline 19 & 10 & 13 & - & - & - & - & - & - & - & - & - & - & - & - & - & - & - \\
\hline 20 & 13 & - & - & - & - & - & - & - & - & - & - & - & - & - & - & - & - \\
\hline
\end{tabular}

NC: non convergence

Table 4. Summary of cases for the sensitivity analysis

\begin{tabular}{|c|c|c|c|c|c|}
\hline Case & Model & Porosity $(\phi)$ & Saturation $\left(S_{r}\right)$ & Density $(\rho)$ & Results \\
\hline 1 & Hyvert & Random field & Constant & $\mathrm{n} / \mathrm{a}$ & \\
& Papadakis & Random field & Constant & $\mathrm{n} / \mathrm{a}$ & \multirow{2}{*}{ Figure 7 } \\
& Miragliotta & Random field & Constant & n/a & \\
& Ying-Yu & Random field & Constant & Constant & \\
\hline 2 & Hyvert & Constant & Random field & $\mathrm{n} / \mathrm{a}$ & \\
& Papadakis & Constant & Random field & $\mathrm{n} / \mathrm{a}$ & \multirow{2}{*}{ Figure 8} \\
& Miragliotta & Constant & Random field & $\mathrm{n} / \mathrm{a}$ & \\
& Ying-Yu & Constant & Random field & Constant & \\
\hline 3 & Ying-Yu & Constant & Constant & Random field & Figure 9 \\
\hline 4 & Hyvert & Random field & Random field & $\mathrm{n} / \mathrm{a}$ & \\
& Papadakis & Random field & Random field & $\mathrm{n} / \mathrm{a}$ & \multirow{2}{*}{ Figure 10 } \\
& Miragliotta & Random field & Random field & $\mathrm{n} / \mathrm{a}$ & \\
& Ying-Yu & Random field & Random field & Random field & \\
\hline
\end{tabular}

$$
\text { n/a: non applicable }
$$


Table 5. Ratio $\hat{\mu}_{b} / b$ for the each model and cases

\begin{tabular}{|c|c|c|c|c|c|c|c|c|c|}
\cline { 3 - 9 } \multicolumn{1}{c|}{} & \multicolumn{2}{c|}{ Hyvert } & \multicolumn{2}{c|}{ Papadakis } & \multicolumn{2}{c|}{ Miragliotta } & \multicolumn{2}{c|}{ Ying-Yu } \\
\cline { 2 - 10 } \multicolumn{1}{c|}{} & $b(\mathrm{~cm})$ & $\hat{\mu}_{b}(\mathrm{~cm})$ & $\hat{\mu}_{b} / b$ & $\hat{\mu}_{b}(\mathrm{~cm})$ & $\hat{\mu}_{b} / b$ & $\hat{\mu}_{b}(\mathrm{~cm})$ & $\hat{\mu}_{b} / b$ & $\hat{\mu}_{b}(\mathrm{~cm})$ & $\hat{\mu}_{b} / b$ \\
\hline \multirow{4}{*}{ Case 1 } & 5 & 9.09 & 1.81 & 9.09 & 1.81 & 8.77 & 1.75 & 9.1 & 1.82 \\
& 50 & 69.2 & 1.38 & 67.9 & 1.35 & 67.6 & 1.35 & 71.9 & 1.43 \\
& 100 & 64.2 & 0.64 & 62.9 & 0.62 & 62.7 & 0.62 & 66.7 & 0.66 \\
\hline \multirow{4}{*}{ Case 2 } & 5 & 9.04 & 1.8 & 9.02 & 1.8 & 9.03 & 1.8 & 9.07 & 1.81 \\
& 50 & 39.8 & 0.79 & 41.9 & 0.83 & 60.2 & 1.2 & 41.1 & 0.82 \\
& 100 & 49 & 0.49 & 52.5 & 0.52 & 63.5 & 0.63 & 39.5 & 0.39 \\
\hline \multirow{3}{*}{ Case3 } & 5 & $\mathrm{n} / \mathrm{a}$ & - & $\mathrm{n} / \mathrm{a}$ & - & $\mathrm{n} / \mathrm{a}$ & - & 20.7 & 4.14 \\
& 50 & $\mathrm{n} / \mathrm{a}$ & - & $\mathrm{n} / \mathrm{a}$ & - & $\mathrm{n} / \mathrm{a}$ & - & 93.1 & 1.86 \\
& 100 & $\mathrm{n} / \mathrm{a}$ & - & $\mathrm{n} / \mathrm{a}$ & - & $\mathrm{n} / \mathrm{a}$ & - & 78.1 & 0.78 \\
\hline \multirow{4}{*}{ Case 4 4} & 5 & 9.09 & 1.81 & 9.11 & 1.82 & 9.13 & 1.82 & 8.91 & 1.78 \\
& 50 & 40.1 & 0.8 & 45.4 & 0.9 & 65.5 & 1.31 & 42 & 0.84 \\
& 100 & 48.2 & 0.48 & 51.7 & 0.51 & 60.2 & 0.6 & 40.2 & 0.4 \\
\hline
\end{tabular}

n/a: non applicable

Table 6. Absolute Error (AE) on the mean of $\hat{b}$ between the Case 4 and all other cases

\begin{tabular}{|c|c|c|c|c|c|c|c|c|c|}
\cline { 3 - 10 } \multicolumn{1}{c|}{} & \multicolumn{2}{c|}{ Hyvert } & \multicolumn{2}{c|}{ Papadakis } & \multicolumn{2}{c|}{ Miragliotta } & \multicolumn{2}{c|}{ Ying-Yu } \\
\cline { 2 - 10 } \multicolumn{1}{c|}{} & $\mathrm{b}(\mathrm{cm})$ & $\hat{\mu}_{b}(\mathrm{~cm})$ & $\mathrm{AE}(\%)$ & $\hat{\mu}_{b}(\mathrm{~cm})$ & $\mathrm{AE}(\%)$ & $\hat{\mu}_{b}(\mathrm{~cm})$ & $\mathrm{AE}(\%)$ & $\hat{\mu}_{b}(\mathrm{~cm})$ & $\mathrm{AE}(\%)$ \\
\hline \multirow{3}{*}{ Case 1 } & 5 & 9.09 & 0 & 9.09 & -0.21 & 8.77 & -3.94 & 9.1 & 2.13 \\
\cline { 2 - 10 } & 50 & 69.2 & 72.56 & 67.9 & 49.55 & 67.6 & 3.2 & 71.9 & 71.19 \\
\cline { 2 - 10 } & 100 & 64.2 & 33.19 & 62.9 & 21.66 & 62.7 & 4.15 & 66.7 & 65.92 \\
\hline \multirow{3}{*}{ Case 2 } & 5 & 9.04 & -0.55 & 9.02 & -0.98 & 9.03 & -1.09 & 9.07 & 1.79 \\
\cline { 2 - 10 } & 50 & 39.8 & -0.74 & 41.9 & -7.7 & 60.2 & -8.09 & 41.1 & -2.14 \\
\cline { 2 - 10 } & 100 & 49 & 1.65 & 52.5 & 1.54 & 63.5 & 5.48 & 39.5 & -1.74 \\
\hline \multirow{3}{*}{ Case 3 3 n/a } & 5 & - & n/a & - & n/a & - & 20.7 & 132.32 \\
\cline { 2 - 10 } & 50 & n/a & - & n/a & - & n/a & - & 93.1 & 121.66 \\
\cline { 2 - 10 } & 100 & n/a & - & n/a & - & n/a & - & 78.1 & 94.27 \\
\hline
\end{tabular}

n/a: non applicable

Table 7. Recommendations about modelling input parameters as random fields for each model

\begin{tabular}{|c|c|c|c|}
\hline Model & Porosity $(\phi)$ & Saturation $\left(S_{r}\right)$ & Density $(\rho)$ \\
\hline Hyvert & R2 & R1 & n/a \\
\hline Papadakis & R2 & R1 & n/a \\
\hline Miragliotta & R1 & R2 & n/a \\
\hline Ying-Yu & R1 & NR & NR \\
\hline
\end{tabular}

R1: recommended (priority), R2: recommended (second priority), NR: non recommended, n/a: non applicable 
Table 8. Summary of study cases for evaluating the recommendations obtained from numerical analysis

\begin{tabular}{|c|c|c|c|c|}
\hline Model & Case & Porosity $(\phi)$ & Saturation $\left(S_{r}\right)$ & Density $(\rho)$ \\
\hline \multirow{3}{*}{ Hyvert } & Full & Random field & Random field & $\mathrm{n} / \mathrm{a}$ \\
& Reduced 1 & Constant & Random field & $\mathrm{n} / \mathrm{a}$ \\
& Reduced 2 & Random field & Constant & $\mathrm{n} / \mathrm{a}$ \\
\hline \multirow{3}{*}{ Papadakis } & Full & Random field & Random field & $\mathrm{n} / \mathrm{a}$ \\
& Reduced 1 & Constant & Random field & $\mathrm{n} / \mathrm{a}$ \\
& Reduced 2 & Random field & Constant & $\mathrm{n} / \mathrm{a}$ \\
\hline \multirow{3}{*}{ Miragliotta } & Full & Random field & Random field & $\mathrm{n} / \mathrm{a}$ \\
& Reduced 1 & Random field & Constant & $\mathrm{n} / \mathrm{a}$ \\
& Reduced 2 & Constant & Random field & $\mathrm{n} / \mathrm{a}$ \\
\hline \multirow{3}{*}{ Ying-Yu } & Full & Random field & Random field & Random field \\
& Reduced 1 & Random field & Constant & Constant \\
& Reduced 2 & Constant & Random field & Constant \\
& Reduced 2 (bis) & Constant & Constant & Random field \\
\hline
\end{tabular}

n/a: non applicable

Table 9. Absolute error (AE) between $\hat{\mu}_{b}$ computed from the models and $\hat{\mu}_{b}$ estimated from the data

\begin{tabular}{|c|c|c|c|c|c|}
\hline & & \multicolumn{2}{|c|}{ Side A } & \multicolumn{2}{|c|}{ Side C } \\
\hline & Case & $\hat{\mu}_{b}(\mathrm{~cm})$ & $\mathrm{AE}(\%)$ & $\hat{\mu}_{b}(\mathrm{~cm})$ & $\mathrm{AE}(\%)$ \\
\hline Measurements & $\mathrm{n} / \mathrm{a}$ & 10.35 & $\mathrm{n} / \mathrm{a}$ & 7.39 & $\mathrm{n} / \mathrm{a}$ \\
\hline \multirow{3}{*}{ Hyvert } & Full & 7.19 & 31 & 9.47 & 28 \\
\hline & Reduced 1 & 6.57 & 37 & 9.5 & 28 \\
\hline & Reduced 2 & 12 & 14 & 8.45 & 14 \\
\hline \multirow{3}{*}{ Papadakis } & Full & 7.89 & 24 & 9.12 & 23 \\
\hline & Reduced 1 & 6.63 & 36 & 9.64 & 30 \\
\hline & Reduced 2 & 12 & 14 & 8.07 & 9 \\
\hline \multirow{3}{*}{ Miragliotta } & Full & 9.18 & 12 & 5.62 & 23 \\
\hline & Reduced 1 & 12.4 & 18 & 4.83 & 34 \\
\hline & Reduced 2 & 10.6 & 0 & 6.24 & 15 \\
\hline \multirow{4}{*}{ Ying-Yu } & Full & 7.21 & 31 & 5.33 & 27 \\
\hline & Reduced 1 & 12.4 & 18 & 5.47 & 25 \\
\hline & Reduced 2 & 10.8 & 2 & 6.71 & 9 \\
\hline & Reduced 2 (bis) & $\mathrm{NC}$ & - & 6.3 & 14 \\
\hline
\end{tabular}

n/a: non applicable 


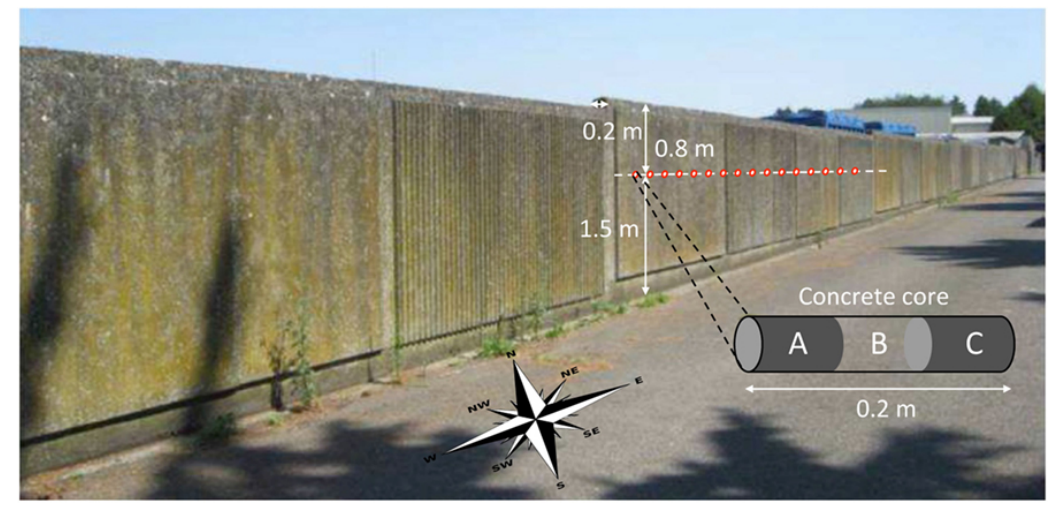

Figure 1. Studied wall
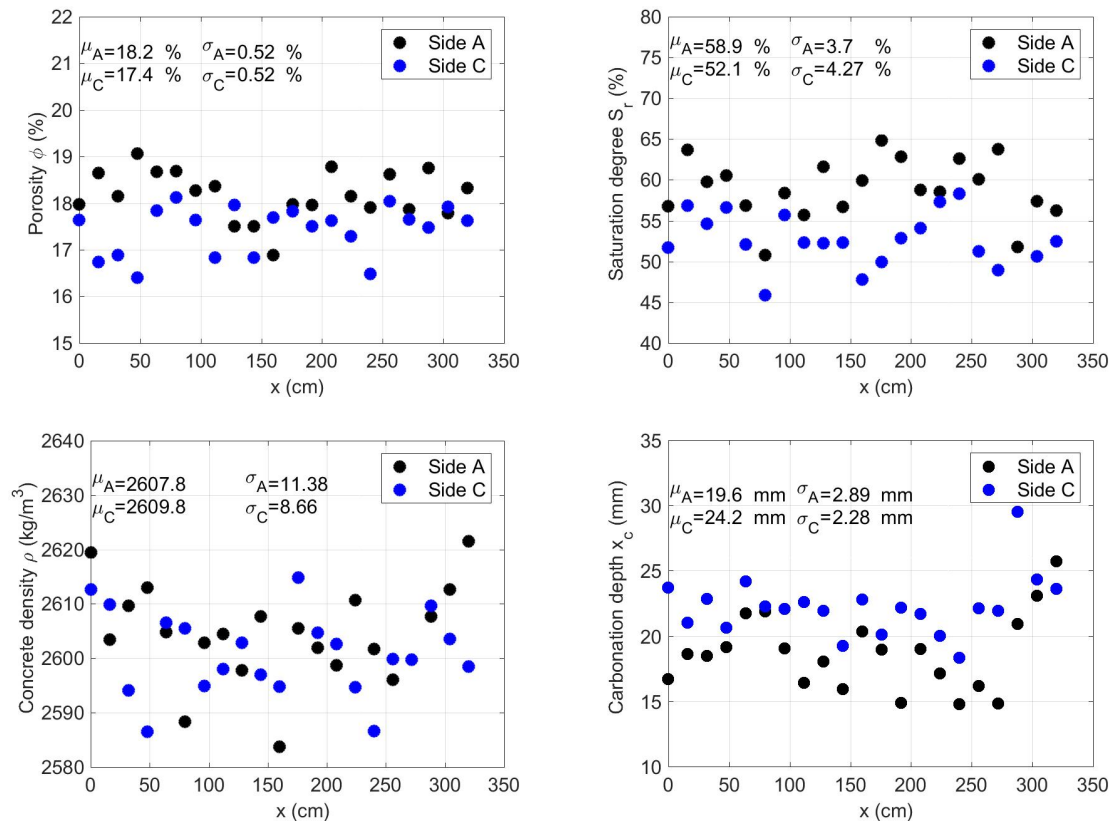

Figure 2. Measurements of porosity, saturation degree, density and carbonation depth 


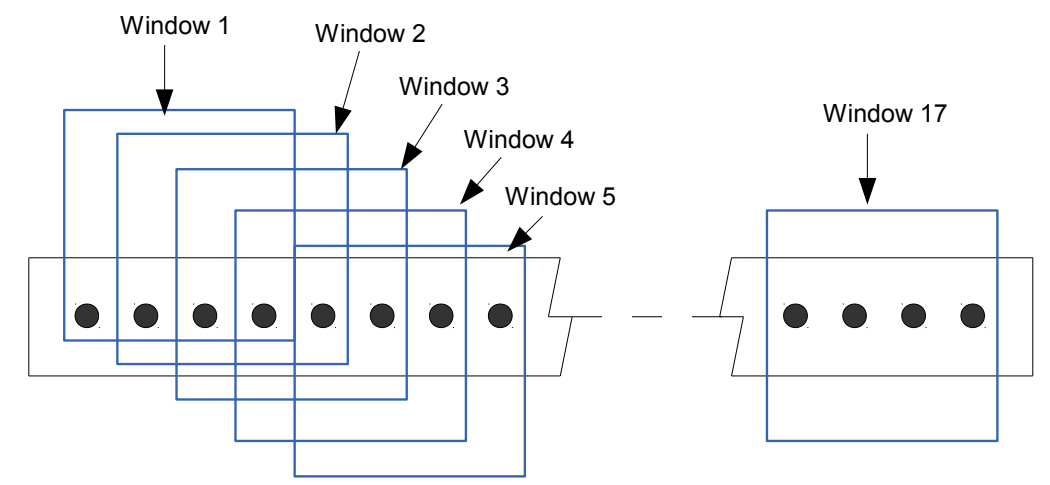

Figure 3. Positions of window with 4 measurements 


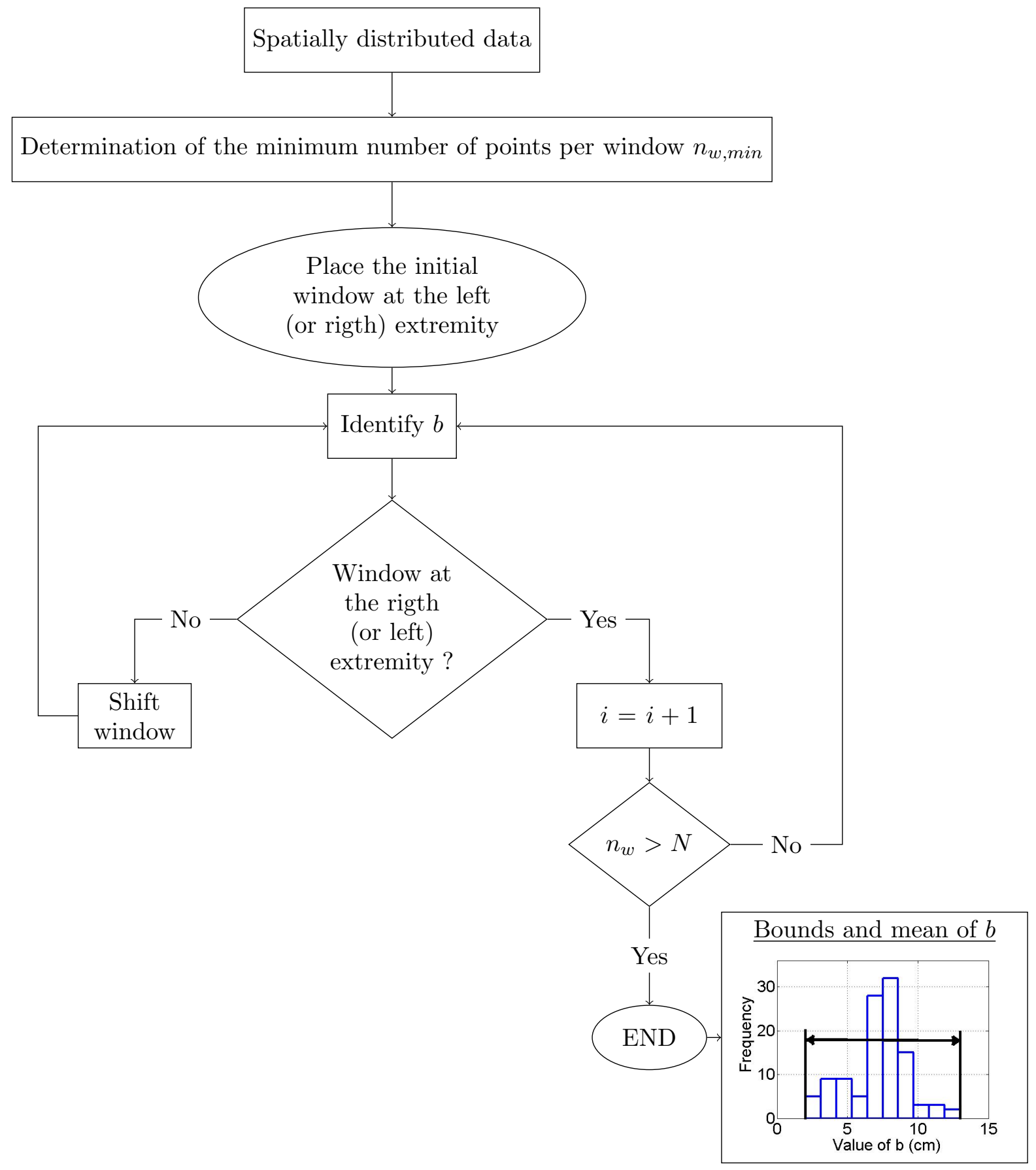

Figure 4. Algorithm of the windowing method 


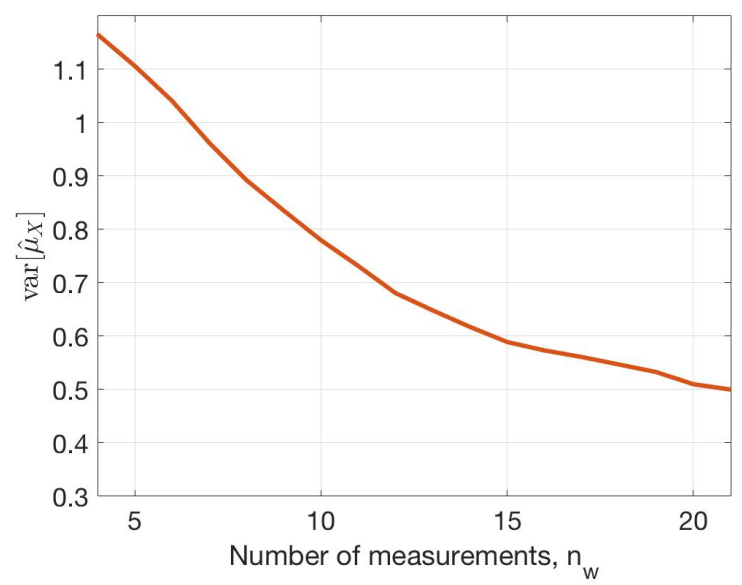

Figure 5. Variance of the mean estimate $\operatorname{var}\left[\hat{\mu}_{X}\right]$ for the carbonation data of side $\mathrm{A}$

Porosity

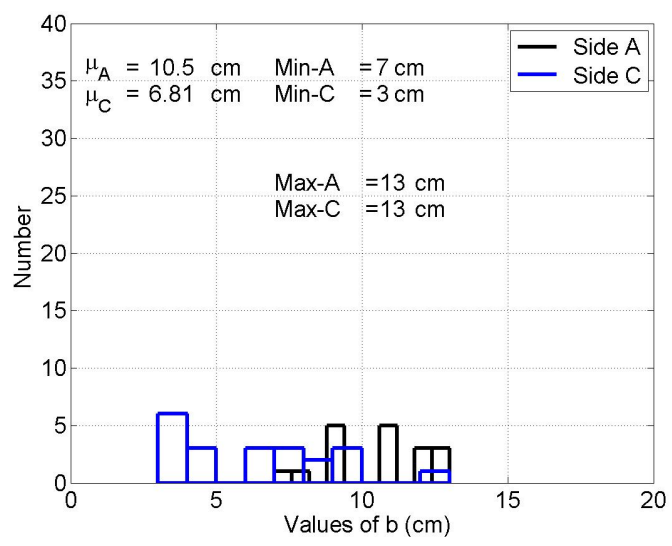

Concrete density

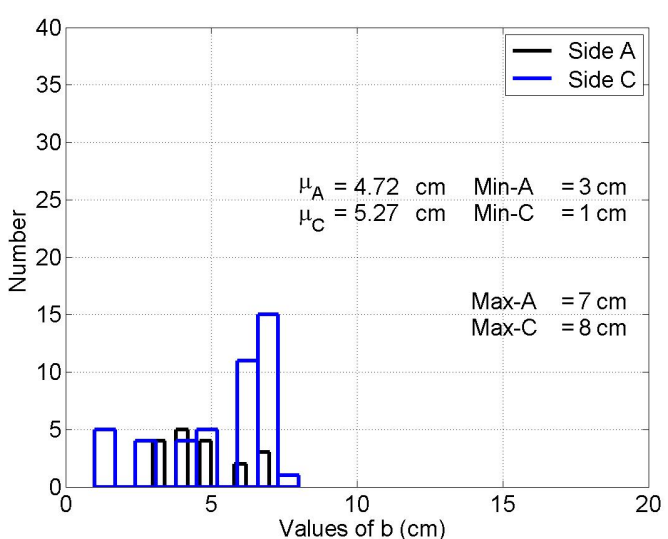

Saturation degree

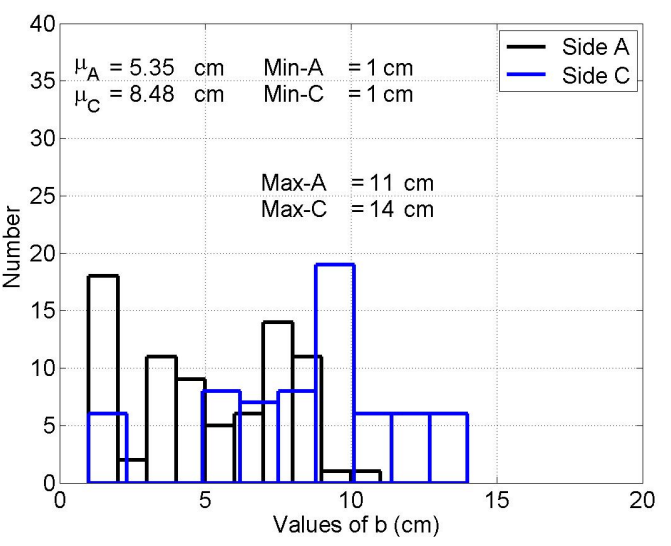

Carbonation depth

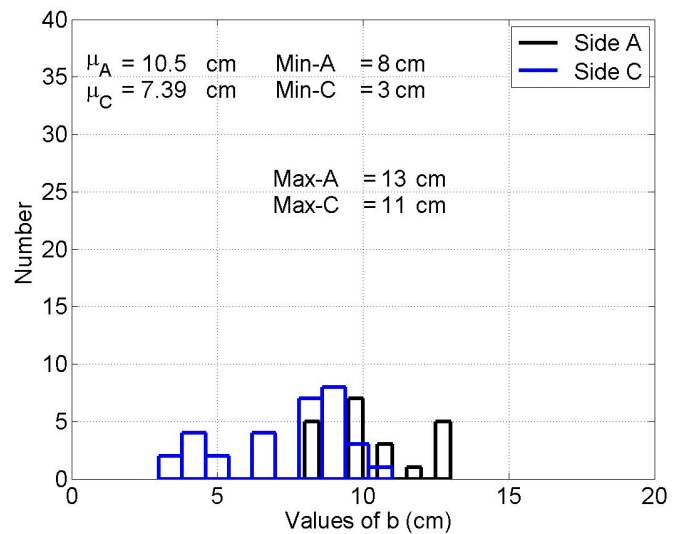

Figure 6. Identified values of $\hat{b}$ for porosity, saturation degree, concrete density and carbonation depth 


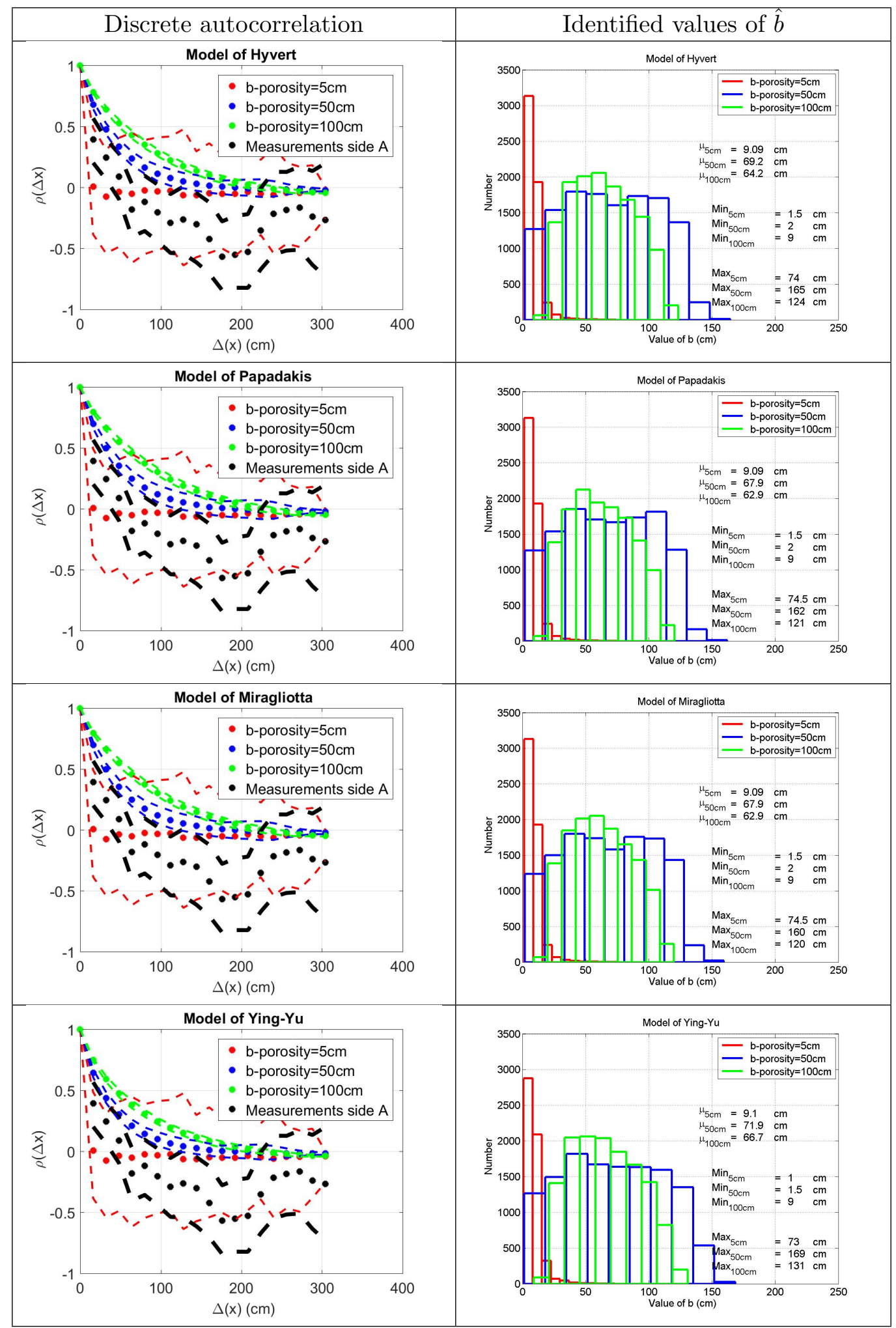

Figure 7. Sensitivity of each model with respect to random field of porosity (Case 1) 


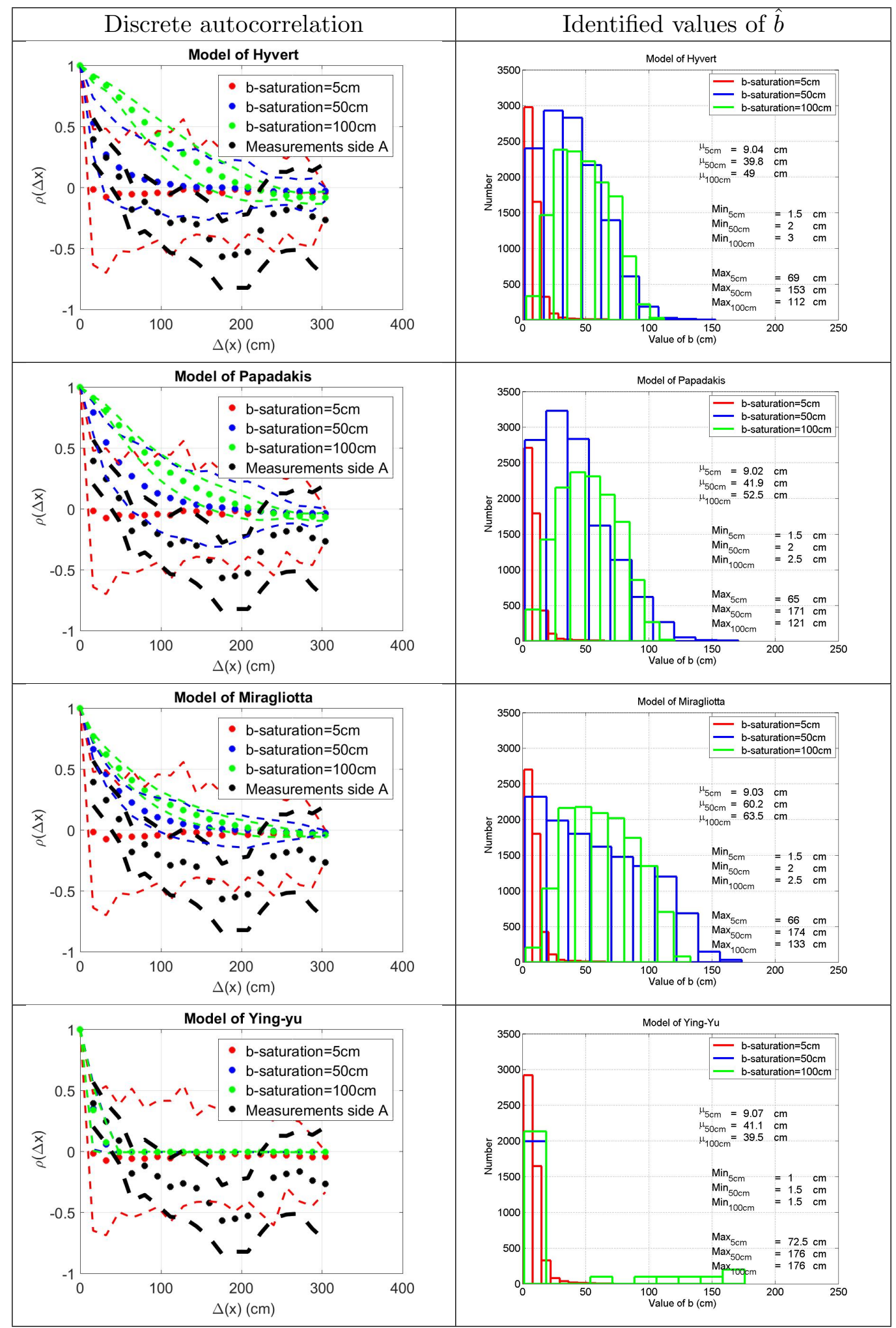

Figure 8. Sensitivity of each model with respect to random field of saturation degree (Case 2) 


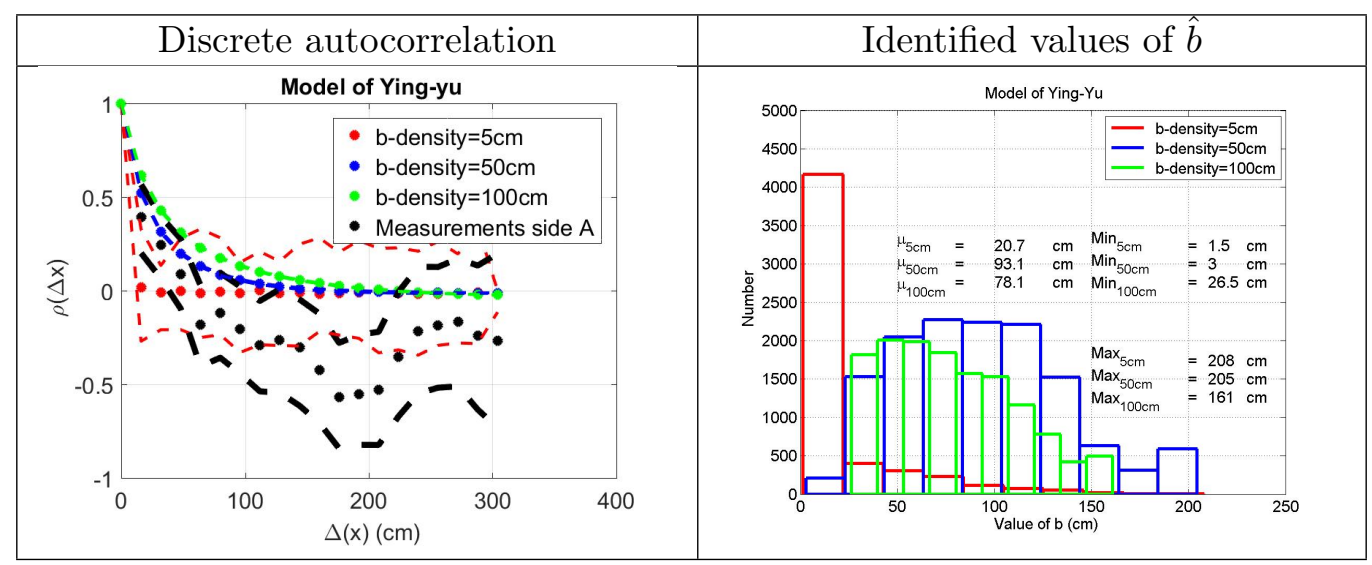

Figure 9. Sensitivity of each model with respect to random field of concrete density (Case 3) 


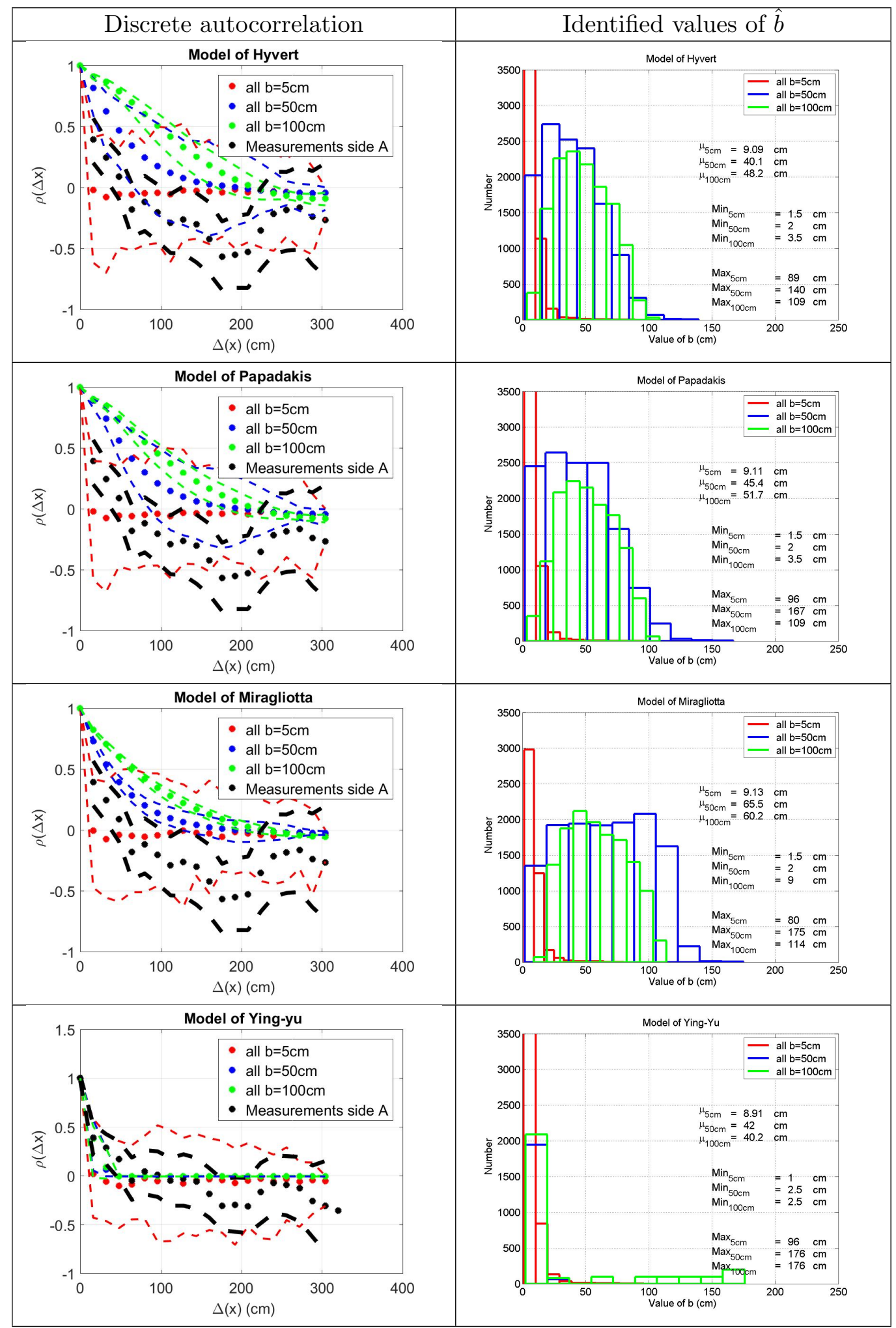

Figure 10. Sensitivity of each model with respect to random field of all inputs (Case 4) 

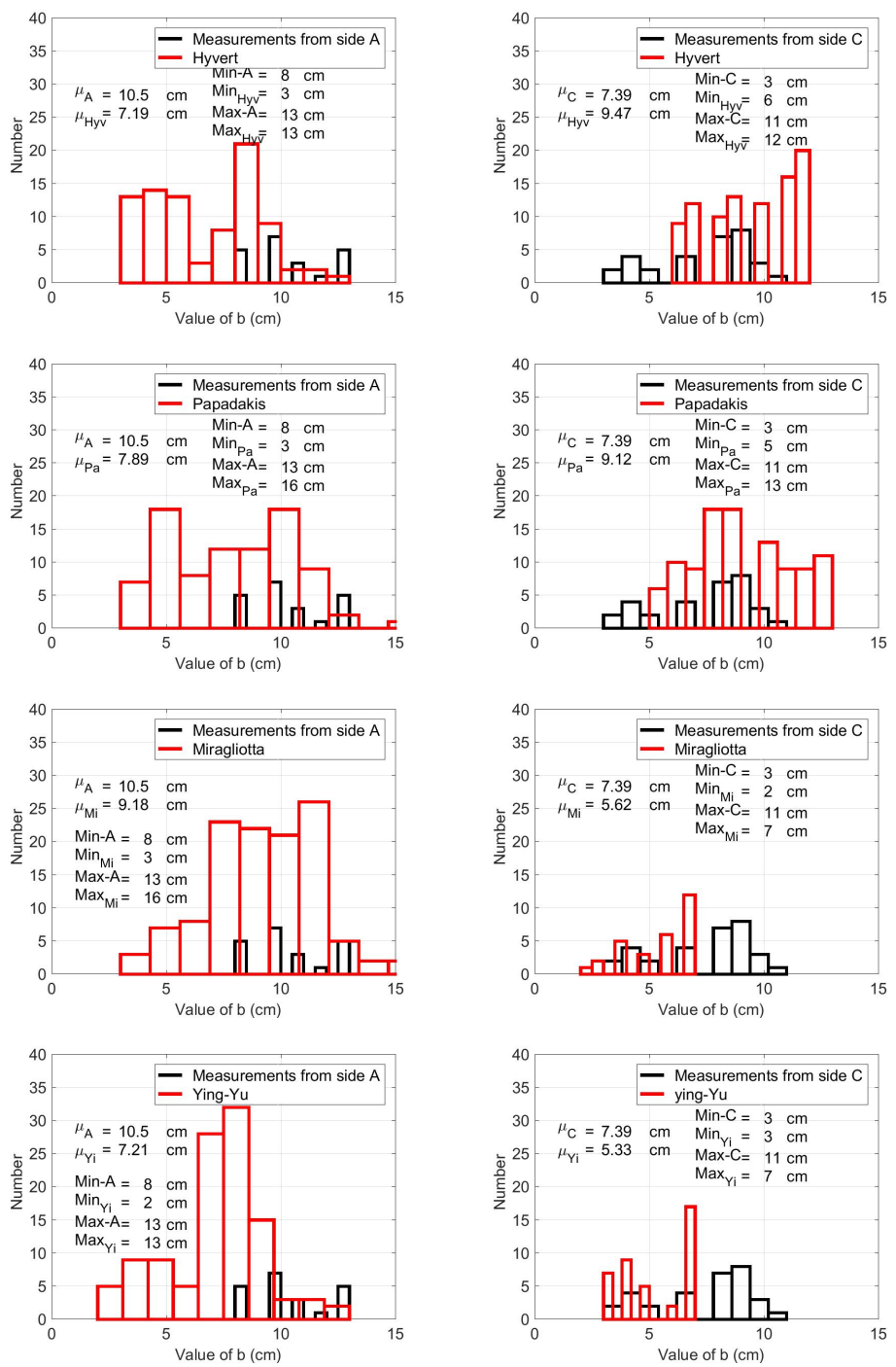

Figure 11. Correspondence between identified values of $\hat{b}$ from models outputs and measurements for Full cases 

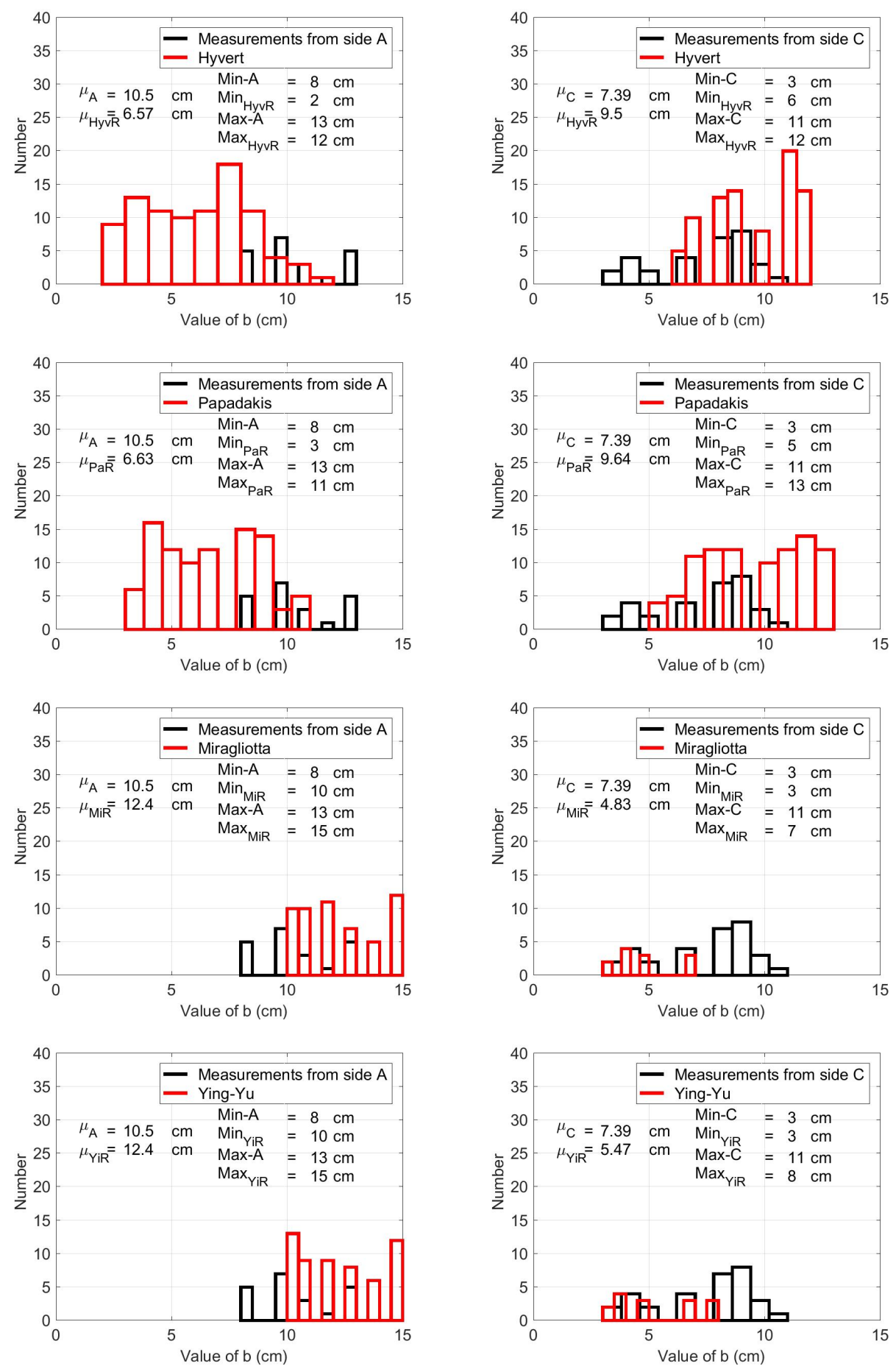

Figure 12. Correspondence between identified values of $\hat{b}$ from models outputs and measurements for Reduced 1 cases 

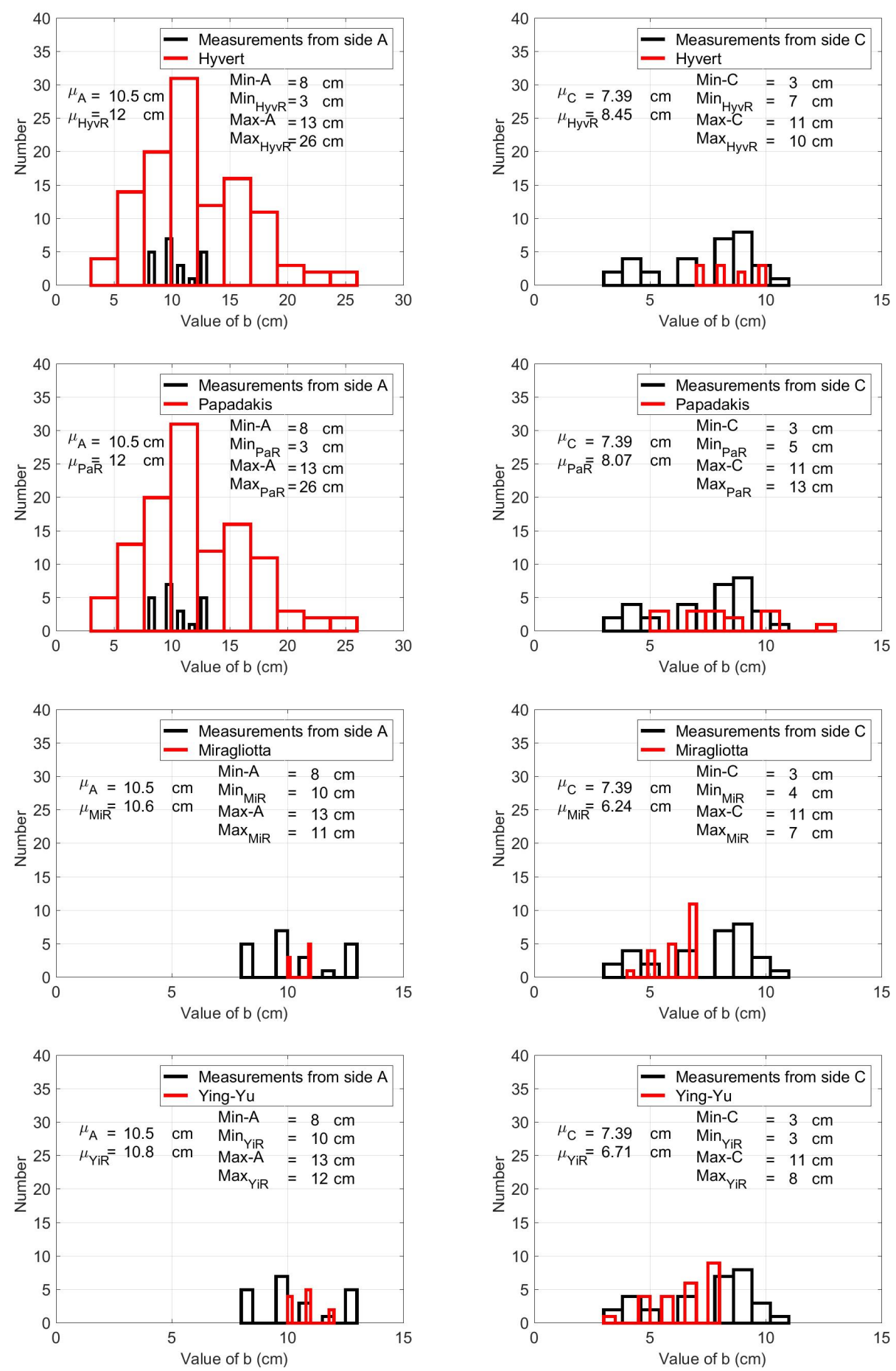

Figure 13. Correspondence between identified values of $\hat{b}$ from models outputs and measurements for Reduced 2 cases 


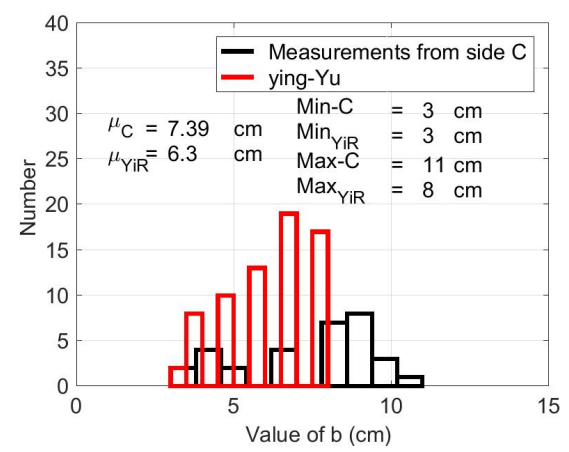

Figure 14. Supplementary correspondence between identified values of $\hat{b}$ from models outputs and measurements for Reduced 2 bis case and the model of Ying-Yu 


\section{Appendix A. Models}

According to (Rakotovao Ravahatra et al., 2019), concrete carbonation models can be written in a generalized expression:

$$
x(t)=\sqrt{k_{\text {exp }} k_{\text {exe }} k_{P} D_{C O_{2}}} \sqrt{t}
$$

where $x(t)[\mathrm{m}]$ is the carbonation depth at time $t[\mathrm{~s}], k_{\exp }$ is a factor which introduces environmental conditions, $k_{\text {exe }}$ is a factor accounting for execution conditions, $k_{P}$ is a factor accounting for the interaction between the diffusion coefficient of the carbon dioxide $D_{\mathrm{CO}_{2}}\left[\mathrm{~m}^{2} / \mathrm{s}\right]$ and the concrete porosity $\phi . k_{P}$ is expressed as:

$$
k_{P}=k_{P, M} k_{P, E}
$$

where $k_{P, M}$ is related to material properties and $k_{P, E}$ to exposure conditions. Expressions of $k_{P, M}, k_{P, E}, k_{e x p}$ and $k_{\text {exe }}$ are given in Table A1 for each considered model. $\alpha_{1}$ and $n_{1}$ are fitting parameters, $f_{p}$ is the volumetric fraction of the cement paste, $R$ is the gas constant (8.31 USI), RH is Relative humidity, $T$ is temperature, $P_{\mathrm{CO}_{2}}$ is carbon dioxide pressure, $\phi$ is porosity, $S_{r}$ is saturation degree, $\rho$ is concrete density, [Component] is the "component" content, $C_{a b s}$ is the absorbed carbone dioxide $\left(C_{a b s}=\phi\left(1-S_{r}\right) \times C_{0}\right), C_{0}$ is the $C_{2}$ content at the exposed surface, $k_{e}$ is a parameter which assesses environmental conditions, $k_{c}$ is a parameter which considers cure conditions.

$$
k_{e}=\left(\frac{1-\left(\frac{\mathrm{RH}}{100}\right)^{2.5}}{1-\left(\frac{\mathrm{RH}_{r e f}}{100}\right)^{2.5}}\right)^{5}
$$

where $\mathrm{RH}_{r e f}$ corresponds to a reference relative humidity $(\simeq 65 \%)$. HR could be computed using $S_{r}$ from desorption curves.

Despite the fact that cement paste hydrates and unhydrates contents are input parameters for the same models, it was decided to consider their variability through hydration degree $\alpha_{h y d}$ and cement content $c$, using the empirical expressions, found in Hyvert (2009). The mean value of measured compressive strength is $40 \mathrm{MPa}$. This value is similar to $\mathrm{C} 45$ concrete. Therefore, we assume a cement content equal to $c=350 \mathrm{~kg} / \mathrm{m}^{3}$. Table A2 presents a cement composition which could suit for such a concrete. The values of the other parameters are given in Table A3. 


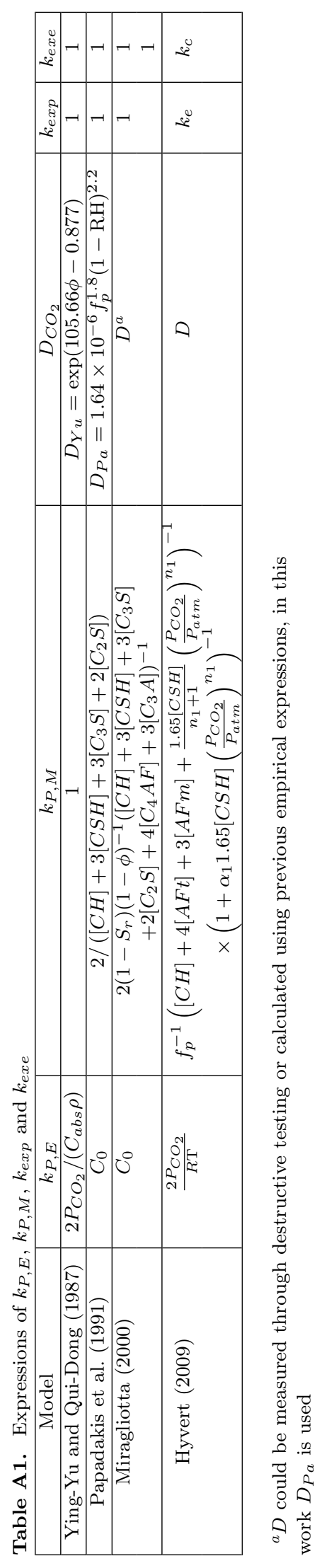


Table A2. Cement composition (\%)

\begin{tabular}{|c|c|}
\hline Component & $(\%)$ \\
\hline $\mathrm{SiO}_{2}$ & 20.1 \\
\hline $\mathrm{Al}_{2} \mathrm{O}_{3}$ & 5 \\
\hline $\mathrm{Fe}_{2} \mathrm{O}_{3}$ & 3 \\
\hline $\mathrm{CaO}$ & 64.1 \\
\hline $\mathrm{MgO}$ & 1 \\
\hline $\mathrm{SO}_{3}$ & 3.2 \\
\hline $\mathrm{K}_{2} \mathrm{O}$ & 0.72 \\
\hline
\end{tabular}

Table A3. Values of input parameters for carbonation models

\begin{tabular}{|c|c|c|}
\hline Parameter & Unit & Value \\
\hline $\mathrm{RH}$ & $\%$ & 72.91 \\
\hline$k_{c}$ & - & 0.63 \\
\hline$k_{t}$ & - & 0.98 \\
\hline $\mathrm{T}$ & $K$ & 284.04 \\
\hline$c$ & $\mathrm{~kg} / \mathrm{m}^{3}$ & 350 \\
\hline$\alpha_{h y d}$ & - & 0.81 \\
\hline$P_{a t m}$ & $\mathrm{~Pa}$ & 101325 \\
\hline$P_{C O_{2}}$ & $\mathrm{~Pa}$ & 40.53 \\
\hline$C_{0}$ & $\mathrm{~kg} / \mathrm{m}^{3}$ & $6.5 \times 10^{-4}$ \\
\hline$\alpha_{1}$ & $\mathrm{~L} / \mathrm{mol}$ & 23.5 \\
\hline$n_{1}$ & - & 0.67 \\
\hline
\end{tabular}

